Research Article

\title{
Taenia crassiceps Antigens Control Experimental Type 1 Diabetes by Inducing Alternatively Activated Macrophages
}

\author{
Arlett Espinoza-Jiménez, Roberto De Haro, and Luis I. Terrazas \\ Unidad de Biomedicina, Facultad de Estudios Superiores Iztacala, Universidad Nacional Autónoma de México, Av. De los Barrios 1, \\ Los Reyes Iztacala, Tlalnepantla 54090, MEX, Mexico \\ Correspondence should be addressed to Luis I. Terrazas; literrazas@unam.mx
}

Received 8 May 2017; Revised 19 August 2017; Accepted 29 August 2017; Published 8 November 2017

Academic Editor: Mirella Giovarelli

Copyright (c) 2017 Arlett Espinoza-Jiménez et al. This is an open access article distributed under the Creative Commons Attribution License, which permits unrestricted use, distribution, and reproduction in any medium, provided the original work is properly cited.

\begin{abstract}
Type 1 diabetes (T1D) is an autoimmune disease caused by the selective destruction of the pancreatic $\beta$-cells, causing inability to produce insulin. Proinflammatory cytokines such as IL- $1 \beta$, IL-6, TNF- $\alpha$, IFN- $\gamma$, IL-12, IL-17, and NO can be released by CD4 and $\mathrm{CD}^{+}$lymphocytes as well as by classically activated macrophages (CAM $\left.\phi s\right)$, which are important in the development of T1D. Helminth infections have been shown to prevent T1D, mainly through Th2-biased responses and increased recruitment of regulatory cell populations. Previously, we have shown that Taenia crassiceps infection in mice significantly reduces hyperglycemia, insulitis, and the incidence of T1D. In this study, we determined whether T. crassiceps-derived products such as soluble (TcS) or excreted/secreted (TcES) antigens might have a beneficial influence on the development of experimental T1D. Treatment with different doses before or after induction of T1D was analyzed. Mice that were pretreated with TcS were unable to develop T1D, whereas those receiving TcES early after T1D induction displayed significantly reduced insulitis and hyperglycemia along with increased recruitment of alternatively activated macrophages (AAM $\phi$ s) and myeloid-derived suppressor cells (MDSCs). Finally, we examined the modulatory role of AAM $\phi s$ on T1D by depleting macrophages with clodronate-loaded liposomes, demonstrating that $\mathrm{AAM} \phi$ s are key cells in T1D regulation.
\end{abstract}

\section{Introduction}

Type 1 diabetes (T1D) is an autoimmune disease caused by the selective destruction of the pancreatic $\beta$-cells, lowering insulin production [1]. The absence of insulin disturbs the regulation of blood glucose concentration, resulting in severe hyperglycemia. The onset of diabetes involves both genetic and environmental factors [2]. Proinflammatory cytokines such as IL- $1 \beta$, IL- 6 , TNF- $\alpha$, IFN- $\gamma$, IL-12, IL-17, and nitric oxide (NO) can be released by $\mathrm{CD}^{+}$and $\mathrm{CD} 8^{+} \mathrm{T}$ lymphocytes as well as by $\mathrm{CAM} \phi$ s, which seems to be important in the development of insulitis and the death of beta cells $[3,4]$. Experimental rodent models such as nonobese diabetic mice (NOD) or multiple low doses of streptozotocin (MLD-STZ) have provided new knowledge and strategies to regulate this disease [5].

During the last few years, anti-inflammatory response has been proposed as a helpful way to regulate the immune system during autoimmune diseases. For example, in NOD mice, the administration of IL-4 or IL-10 may prevent the development of diabetes [6]. In addition, helminth infections have been shown to prevent T1D development. The mechanisms of this effect may consist of inducing a strong Th2-type response and increasing regulatory cell populations, such as Treg, MDSCs, and AAM $\phi$ s, that exert regulatory effects on the immune system of their host [7]. Infections with Schistosoma mansoni [8], Heligmosomoides polygyrus [9, 10], Litomosoides sigmodontis [11], Trichinella spiralis [9], Nippostrongylus brasiliensis, and Strongyloides venezuelensis [12] can provide different levels of protection against the onset of T1D in NOD and MLD-STZ-induced T1D mice, but all these parasites may induce dangerous side effects in their hosts. For example, Schistosoma mansoni, Litomosoides sigmodontis, and Trichinella spiralis have complex life cycles that include migration through several tissues including lungs, bladders, and muscle where these 
parasites cause damage. Previously, we have shown that infection with the cestode Taenia crassiceps significantly decreases hyperglycemia, insulitis and, consequently, the incidence of T1D in mice treated with MLD-STZ. Moreover, higher levels of IL-4 in sera and an increase in the $\operatorname{AAM} \phi$ population were induced as well, suggesting that this population could be important in protection against T1D [13].

$\operatorname{AAM} \phi s$ are induced by Th2 responses, such as those induced by helminth infections [14]. AAM $\phi$ s can produce IL-10 and TGF- $\beta$ and downregulate levels of proinflammatory cytokines. Additionally, AAM $\phi$ s express suppressive molecules such as PDL-1 and PDL-2 (PD-1 ligands), which are both associated with inhibiting proliferative responses [15-17]. Likewise, AAM $\phi$ s express arginase-1, which induces a shift in arginine metabolism towards the production of Lornithine, a precursor for polyamines and collagen, important molecules in wound healing $[18,19]$. In contrast, $\mathrm{CAM} \phi s$ produce iNOS, which converts L-arginine into ROS and NO. Free radicals and NO are critical in inducing damage to $\beta$-cells $[15,19]$. Interestingly, in many studies, infection with helminths or treatment with their antigens led to an increase in the numbers of $\operatorname{AAM} \phi s$. For example, S. mansoni infection or treatment with soluble worm antigen (SWA) or soluble egg antigen (SEA) brings about a reduction in the inflammatory response in NOD mice, which has been correlated with increasing $\operatorname{AAM} \phi$ and Treg populations [20-22]. In another study, E/S products from Fasciola hepatica (FhES) reduced pancreatic islet damage and hyperglycemia in NOD mice, increasing $\operatorname{AAM} \phi s$ in the pancreas and pancreatic lymph nodes [23]. Furthermore, adoptive transfer of $\mathrm{AAM} \phi$ s induced in vitro by $\mathrm{IL}-4$ and IL-13 in NOD mice decreased hyperglycemia and insulitis, suggesting a protective effect in this population [24]. Similarly, another study using the MLD-STZ model revealed that angiogenesis and the wound healing process were more effective if $\mathrm{AAM} \phi$ s were present [25]. However, in most of these studies, helminths or their products were administered before T1D onset, leaving open the question of whether such treatments may be useful as a therapeutic alternative.

Another important population also able to suppress immune responses is the myeloid-derived suppressor cell (MDSC) population. MDSCs are a heterogeneous population of myeloid progenitor cells and immature myeloid cells that delay their maturation and can be differentiated by the expression of different markers such as CD11b and Gr1 [26]. Two subsets of MDSCs were recently defined: monocytic MDSCs, which express CD11b ${ }^{+}$Ly6$\mathrm{C}^{\text {high }} \mathrm{Ly} 6 \mathrm{G}^{-}$, and granulocytic MDSCs, which express

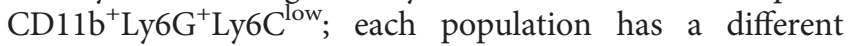
function in cancer, parasitic infections, and autoimmunity $[26,27]$. However, their role in T1D development is largely unknown.

The purpose of this study was to determine whether soluble or excreted/secreted products of $T$. crassiceps could protect against T1D induced by MLD-STZ. Additionally, we evaluated the protective role of $\operatorname{AAM} \phi s$ and MDSCs induced by T. crassiceps and their products in T1D development.

\section{Materials and Methods}

2.1. Mice. Six- to eight-week-old male BALB/cAnN mice were purchased from Harlan Laboratories (Mexico) and were maintained in a pathogen-free environment at the animal facility at FES-Iztacala, UNAM, in accordance with institutional and national guidelines.

2.2. Parasites and Antigen Preparation/Quantification. Metacestodes of Taenia crassiceps were harvested in sterile conditions from the peritoneal cavity of male BALB/cAnN mice after 2-4 months of infection and were washed 4 times with ice-cold, sterile PBS.

(i) T. crassiceps soluble antigen (TcS) was prepared by homogenizing whole metacestodes ( $10 \mathrm{ml}$ volume) in 2 rounds of 3 seconds each by using a homogenizer (Polytron, Kinematica). The homogenates were centrifuged at $2000 \times \mathrm{g}$ for 20 minutes at $4^{\circ} \mathrm{C}$, and the supernatants, which contained PBS-soluble antigens, were collected and frozen at $-80^{\circ} \mathrm{C}$ until further use. Protein concentration was determined using a Bradford protein assay kit (BioRad). Preparation of TcS was similar to that described in [28].

(ii) $T$ crassiceps excreted/secreted products (TcES) were prepared as described elsewhere by Terrazas et al. [29]; briefly, metacestodes were harvested in sterile conditions following 3 washes with sterile PBS. Metacestodes were seeded in 6-well plates (Costar, Cambridge, MA) for 24 hours at $37^{\circ} \mathrm{C}$ and $5 \% \mathrm{CO}_{2}$. Supernatants were collected and centrifuged at $1000 \times \mathrm{g}$ for 10 minutes. Subsequently, the upper fraction was concentrated using $50 \mathrm{kDa}$ Amicon Ultra Filter tubes (Millipore) and further centrifuged at $2000 \times \mathrm{g}$ for 30 minutes. Protease inhibitors were added to the $\geq 50 \mathrm{kDa}$ fraction, and samples were stored at $-80^{\circ} \mathrm{C}$ until further use. Protein concentration was determined using a Bradford protein assay kit (BioRad).

2.3. Treatments. Different experimental groups as detailed in Table 1 were used.

2.4. Blood Glucose Monitoring. Blood glucose was measured at $0,1,3$, and 6 weeks after T1D induction using an AccuCheck Advantage glucometer (Roche Diagnostics) in animals that had been fasted for 6 hours. Animals were considered diabetic when fasting blood glucose was greater than $200 \mathrm{mg} / \mathrm{dl}$.

2.4.1. Glucose Tolerance Test. At 6 weeks after induction of $\mathrm{T} 1 \mathrm{D}$, mice were subjected to an intraperitoneal glucose tolerance test in order to establish the effects of their diabetes on glucose metabolism. The mice were fasted for 6 hours prior to sample collection. A basal blood sample (time 0) was collected by tail snip, and plasma glucose was evaluated using an Accu-Check Advantage glucometer. Mice were injected i.p. with filtered D-glucose $(1.5 \mathrm{mg} / \mathrm{kg})$. Glucose levels were evaluated again at the 30-, 60-, and 120-minute time points. 
TABLe 1: Description of experimental groups.

\begin{tabular}{llc}
\hline Treatment & Description & Groups \\
\hline \multirow{3}{*}{ STZ } & $\begin{array}{l}\text { Diabetic mice were induced with multiple low doses of streptozotocin (MLD-STZ). They received during five days } \\
\text { consecutive intraperitoneal injections (i.p) of STZ (Sigma-Aldrich; } 45 \mathrm{mg} / \mathrm{kg}) \text { dissolved in } 0.1 \mathrm{M} \text { sodium citrate, } \\
\text { pH } 4.5[30]\end{array}$ \\
\hline
\end{tabular}

Mice with MLD-STZ and injections of T. crassiceps soluble antigens

With $50 \mu \mathrm{g}$ of TcS i.p. 3 times for a week, one week before, and during the week of induction with MLD-STZ

Treated constantly with $50 \mu$ g i.p. 3 times per week, one week before, during treatment with MLD-STZ, and for 6 weeks post induction until euthanasia

Treated with $50 \mu$ g i.p. 3 times per week, starting 1 week post induction of T1D and continuing until sacrifice

$\mathrm{STZ} / \mathrm{TcS}-2$

Treated with $100 \mu$ g i.p. 3 times per week during 1 week post induction of T1D and for 6 weeks afterward

Treated with $200 \mu$ g i.p. 3 times per week, starting 1 week post induction T1D and continuing for 6 weeks

Animals treated with injections of T. crassiceps excreted/secreted antigens with MLD-STZ

Treated with $50 \mu$ g i.p. 3 times per week, one week before, during treatment with MLD-STZ, and for 6 weeks post induction

STZ/TcS-5

Animals treated with injections of T. crassiceps excreted/secreted antigens with MLD-STZ
Treated with $50 \mu \mathrm{g}$ i.p. 3 times per week, one week before, during treatment with MLD-STZ, and for 6 weeks
post induction

Treated with $200 \mu$ g i.p. 3 times per week, started one week after induction of T1D and during 6 weeks

$\mathrm{STZ} / \mathrm{TcES}-4$

Six- to eight-week-old mice were infected i.p. with 20 cysticerci, and then we waited 6 weeks post infection to induce diabetes by MLD-STZ. We chose this time period to induce diabetes because we know by a previous report from our group that the change in the immune response to a Th2 response and the appearance of AAM $\phi$ s have been established to occur in the sixth to eighth week post infection [31]. Macrophages were depleted in vivo using dichloromethylene diphosphonate (clodronate) encapsulated in liposomes. Treatment of liposome injections was followed as Reyes et al. [31]

Liposomes

In the second week after T1D-induction, mice were treated with i.p. injections of clodronate liposomes $(\mathrm{Cl})$ or PBS liposomes ( $200 \mu \mathrm{l} /$ mouse i.p. 3 times/week) for 5 weeks post T1D induction

Two weeks after T1D induction by MLD-STZ, T. crassiceps-infected and T. crassiceps-uninfected mice were injected i.p. with $\mathrm{Cl}$ liposomes and PBS liposomes ( $200 \mu \mathrm{l} /$ mouse i.p. 3 times/week) for 5 weeks post T1D induction

$\mathrm{STZ} / \mathrm{Tc} \mathrm{Cl}$

STZ/Tc PBS

An equivalent treatment with clodronate liposomes was done in TcSA-treated Balb/c mice. Animals were injected STZ/TcS Cl on a similar schedule to STZ/TcS-2, the second week before T1D induction and continuing for 4 weeks post STZ/TcS induction. Blood glucose levels were measured for 4 weeks

Untreated Receiving neither antigens nor MLD-STZ

Untreated

2.4.2. Histology. Pancreases from all groups were collected 6 weeks after the induction of diabetes. The tissues were processed and embedded in paraffin, and $5 \mu \mathrm{m}$ sections were cut for analysis. Thin sections were stained with hematoxylin and eosin (H\&E) and evaluated microscopically for the presence of insulitis using the following scoring system: noninfiltrated (healthy islets), peri-insulitis (lymphocytes at the periphery of the islets), insulitis $20 \%$ (insulitis into the interior of the islets $\leq 20 \%$ ), and insulitis $40 \%$ (insulitis into the interior of the islets $\geq 40 \%$ with damage to islet architecture). The insulitis evaluation shown is representative of 10 mice per group (at least 100 islets).

2.4.3. Cytokine ELISAs. Peripheral blood was collected from tail snips at 1, 3, and 6 weeks post induction. Serum IL-4 and TNF- $\alpha$ were measured by sandwich ELISA using a commercial kit purchased from Peprotech, Mexico.

2.4.4. Flow Cytometry. Peritoneal exudate cells (PECs) were obtained from the peritoneal cavity from distinct groups of mice 6 weeks p.i. of MLD-STZ. The cells were washed twice with physiological saline solution, and the red blood cells were lysed by resuspending the cells in Boyle's solution ( $0.17 \mathrm{M}$ Tris and $0.16 \mathrm{M}$ ammonium chloride). Following two washes, the viable cells were counted by trypan blue exclusion with a Neubauer hemocytometer. The PECs were adjusted $\left(1 \times 10^{6}\right.$ cells), and Fc receptors were blocked with anti-mouse CD16/CD32 (Biolegend, CA, USA) and then stained with APC-F4/80, APC-CD11b, FITC-CD11c, FITCMMR, PE-PDL-1, PE-PDL-2, PE-IL-4R $\alpha$, and PE-Gr1 (all from Biolegend, CA, USA) and incubated for 30 minutes at $4^{\circ} \mathrm{C}$ in FACSFlow staining buffer (Becton Dickinson). The cells were analyzed using a FACsCalibur and CellQuest Software (Becton Dickinson).

2.4.5. Statistical Analysis. Data were tested for statistical significance using GraphPad Prism software (version 5.0; Graphpad Software, San Diego, CA, USA). For comparisons between the two groups, the Mann-Whitney $U$ test was applied to test differences with nonparametric data. Experiments with multiple groups were tested by the Kruskal-Wallis test followed by Dunn's multiple comparison 
test. Data are presented as mean \pm SEM; $p$ value $<0.05$ was considered statistically significant $\left({ }^{*} p \leq 0.05,{ }^{* *} p \leq 0.01\right.$, and $\left.{ }^{* * *} p \leq 0.001\right)$.

\section{Results}

3.1. T. crassiceps Soluble Antigen Reduces Experimental T1D in Mice Only If the Treatment Remains Constant. Our first goal was to test whether soluble $T$. crassiceps antigens may protect against T1D through prior or constant exposure. Thus, mice were treated i.p. with $50 \mu \mathrm{g}$ of TcS 3 times a week, starting one week before T1D induction and continuing for six consecutive weeks post T1D induction until euthanasia (STZ/TcS-2), or with a dose of $50 \mu \mathrm{g}$ of soluble antigen one week before induction of MLD-STZ, 3 times in a week (STZ/TcS-1) (Figure 1(a)). Blood glucose was measured once a week. Figure 1(b) shows that STZ/TcS-2 mice had lower blood glucose levels than STZ at 3 and 6 weeks after disease induction; however, STZ/TcS-1 did not present this effect. Surprisingly, STZ/TcS-2 mice, which had a constant exposure to TcS antigen, downregulated the hyperglycemia at borderline of nonpathological levels $(200 \mathrm{mg} / \mathrm{dl})$. Moreover, while the percentage of mice free of diabetes in the STZ group was $0 \%$ (with diabetes defined as glucose levels $>200 \mathrm{mg} / \mathrm{dl}$ ), the STZ/TcS-2 group displayed a significant reduction in T1D (50\%), whereas STZ/TcS-1 had a positive effect in only $25 \%$ of mice (Figure 1(c)). Additionally, we evaluated glucose tolerance in these mice by injecting them i.p. with D-glucose $(1.5 \mathrm{mg} / \mathrm{kg})$, and their blood glucose was evaluated at different times. Hyperglycemia was significantly reduced in mice receiving TcS constantly compared with the STZ and STZ/ TcS-1 groups, which showed hyperglycemia up to $300 \mathrm{mg} / \mathrm{dl}$ (Figure 1(d)).

3.2. Constant Exposure to TcS Reduces Insulitis in T1DInduced Mice. At week 6 post T1D induction, mice were euthanized, and their pancreases were removed and processed for histology to evaluate insulitis. The STZ and STZ/ TcS-1 groups revealed extensive and severe peri-insulitis and insulitis at the islets of Langerhans, with clear loss of islet architecture, while mice constantly exposed to TcS antigen (STZ/TcS-2) displayed a significant reduction in the number of infiltrated islets and the structure remained unmodified in most of them (Figures 2(a) and 2(b)).

3.3. Constant TcS Treatment in Diabetic Mice Induces High Levels of IL-4. To evaluate whether TcS treatment might be able to modify inflammatory response, circulating cytokine levels in sera of the different groups were measured. TNF- $\alpha$, an important cytokine related to pancreatic islet damage, displayed similar levels between groups, but at the sixth week after T1D induction, we observed lightly increasing levels of TNF- $\alpha$ in STZ mice (Figure 3(a)). In contrast, IL-4 was significantly elevated in STZ/TcS-2 mice since the first week of treatment, whereas STZ and STZ/TcS-1 mice displayed lower levels of this cytokine (Figure 3(b)).

3.4. Constant TcS Exposure Recruits AAM $\phi$ s. Next, we examined whether $\mathrm{TcS}$ exposure might favor the recruitment of $\mathrm{AAM} \phi$ s. PECs were analyzed by flow cytometry, and we evaluated $\mathrm{FSC}^{\text {high }} \mathrm{SSC}^{\text {high }} \mathrm{F} 4 / 80^{+}$cells to look for AAM $\phi$ markers. As shown in Figure 4, STZ-treated and STZuntreated mice had reduced percentages of AAM $\phi$ s, while STZ/TcS-2 displayed elevated percentages of AAM $\phi s$, given that the expression levels of MMR, IL- $4 \mathrm{R} \alpha, \mathrm{PDL}-1$, and PDL-2 were significantly upregulated in STZ/TcS-2-treated mice compared to STZ-treated mice.

3.5. Treatment with TcES Reduces the Incidence of T1D. A major question was whether exposure to T. crassiceps products early after T1D induction could be effective to protect diabetic mice. We used 3 different variables with the same treatment regime as described before: mice were injected i.p. with $50 \mu \mathrm{g}$ (STZ/TcS-3), $100 \mu \mathrm{g}$ (STZ/TcS-4), or $200 \mu \mathrm{g}$ (STZ/TcS-5) of TcS started in the first week post T1D induction (Figure 5(a)). In addition, we tested TcES treatment in a constant form, $50 \mu \mathrm{g} /$ dose for 1 week before and 6 weeks after induction of T1D (STZ/TcES-1) or as a post induction treatment 50,100 , or $200 \mu \mathrm{g}$ beginning after the first week post T1D induction (STZ/TcES-2, STZ/TcES-3, and STZ/TcES-4, resp.) using the scheme described before (Figure 5(a)). We found that TcS treatment did not show a protective effect at any dose when the animals were exposed to $T$. crassiceps-derived antigens one week post induction of diabetes (Figure 5(b)). Also, constant TcES (STZ/TcES-1) treatment was insufficient to reduce hyperglycemia. In contrast, a $200 \mu \mathrm{g} /$ mouse dose of TcES antigen (STZ/TcES-4) was more effective to reduce the high levels of glucose in diabetic mice in a post induction scheme (Figure 5(c)). Additionally, the percentage of mice free of diabetes in the STZ group at the 6th week was zero, whereas in STZ/TcES-4, the percentage was 50\%, as in the STZ/TcS-2 (Figure 5(d)).

Next, we evaluated both insulitis in the pancreas and the damage score in the islets. Figures 5(e) and 5(f) show that the STZ/TcES-4 group had significantly less damage in the pancreas and therefore a lower damage score; most of the islets showed neither infiltration nor peri-insular infiltration, as opposed to the STZ group, which displayed severe damage in the islets and developed insulitis in $20-40 \%$.

STZ/TcES-4 treatment also significantly increased the expression of AAM $\phi$ markers such as PDL-2 and MMR compared to the levels in STZ-induced mice (Figure 6(a)).

3.6. Exposure to T. crassiceps-Derived Products after T1D Induction Increases the Population of FSC ${ }^{\text {high }} \mathrm{Gr} 1^{+} \mathrm{CD} 11 b^{+}$ Cells. To examine whether exposure to $T$. crassiceps antigens that were effective in decreasing hyperglycemia (STZ/TcS-2 and STZ/TcES-4) in diabetic mice could modify the recruitment of MDSCs, we evaluated the expression of CD11b and Gr1 markers in peritoneal cells by flow cytometry. As shown in Figure 6(b), STZ/TcS-2 and STZ/TcES-4 treatment led to the recruitment of significantly higher percentages of $\mathrm{FSC}^{\text {high }} \mathrm{SSC}^{\text {high }} \mathrm{CD} 11 \mathrm{~b}^{+} \mathrm{Grl}^{+}$cells $(34 \% \pm 7$ and $75 \% \pm 3$, resp.), compared with STZ mice $(3 \% \pm 0.9)$ and untreated mice $(1.7 \pm 0.07 \%)$. These results suggest a possible positive role for the $\mathrm{CD}_{1} 1 \mathrm{~b}^{+} \mathrm{Gr} 1^{+}$population as well as $\mathrm{AAM} \phi$ s in regulating $\mathrm{T} 1 \mathrm{D}$ development. 


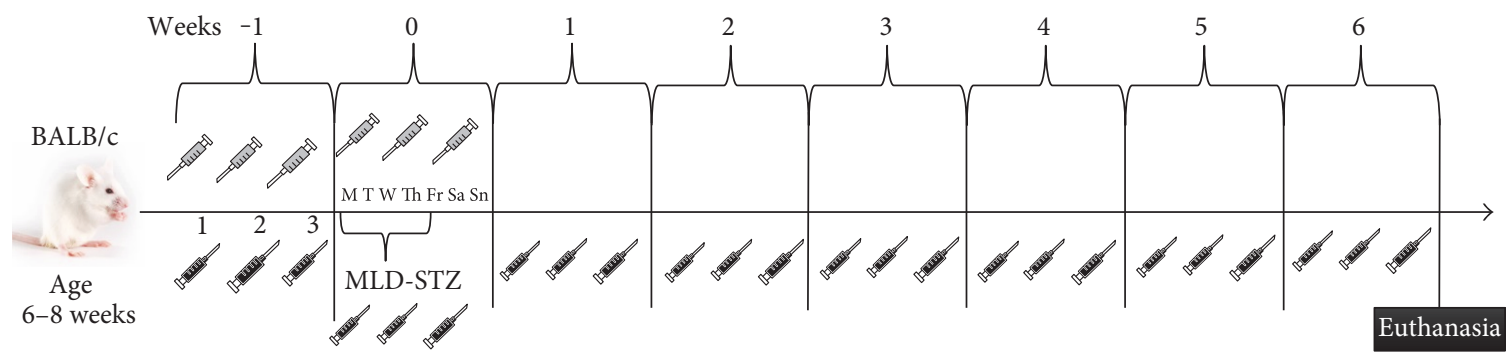

Injection of TcS-1

Injection of TcS-2

(a)

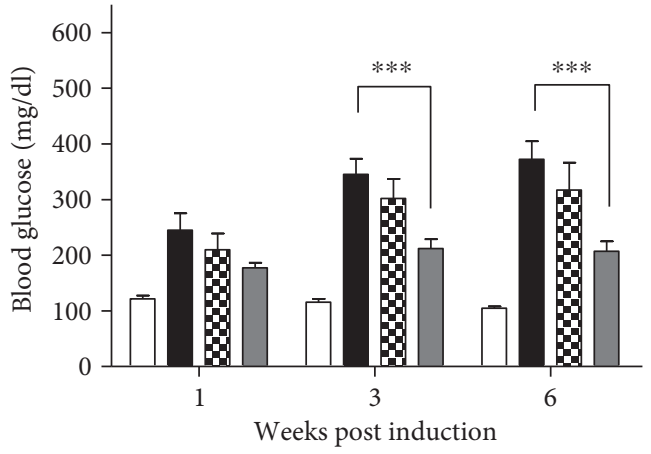

$\square$ Untreated
STZ
STZ/TcS-1

STZ/TcS-2

(b)

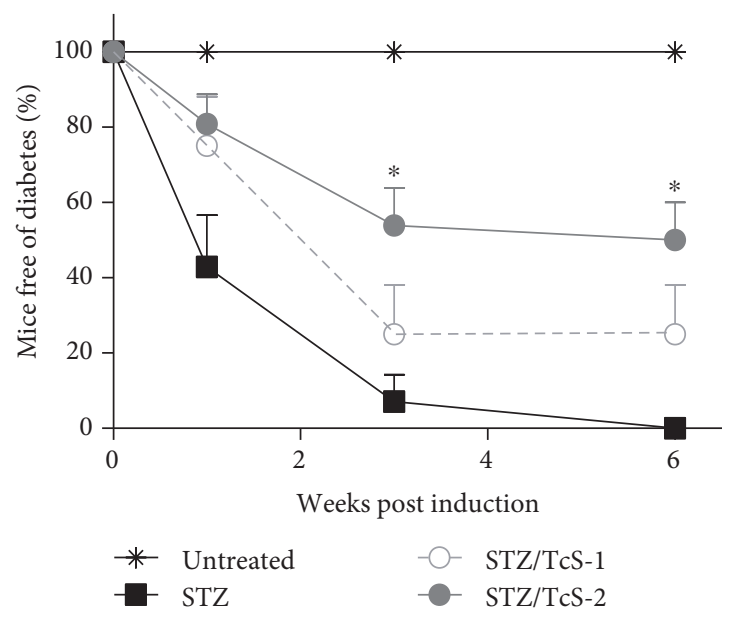

(c)

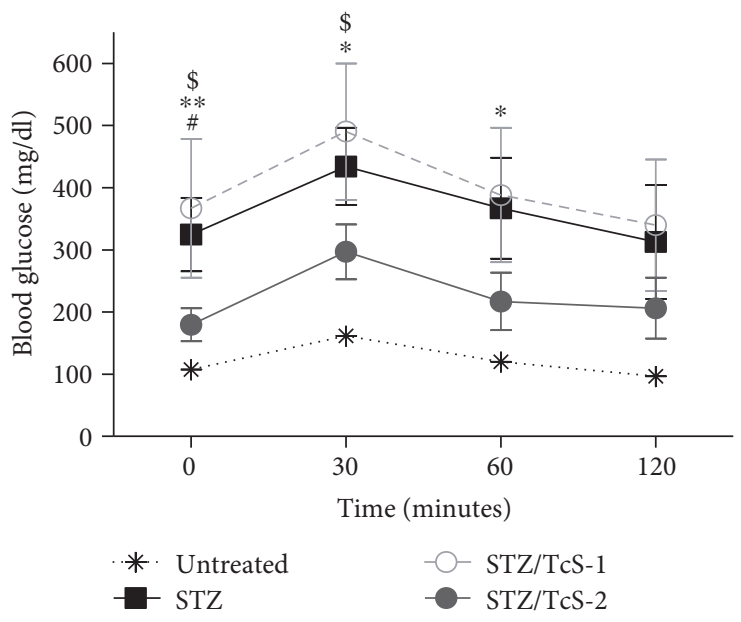

(d)

FIgURE 1: T. crassiceps soluble antigens were protective against T1D development only when antigen was constantly injected (STZ/TcS-2). (a) Methodology diagram illustrating treatment with T. crassiceps antigen. (b) Blood glucose levels for all groups. STZ/TcS-2 treatment was able to significantly reduce glycemia to normal levels $(\leq 200 \mathrm{mg} / \mathrm{dl})$ compared with STZ group. (c) Percentage of mice free of diabetes; mice with glycemia higher than $200 \mathrm{mg} / \mathrm{dl}$ were considered diabetic mice, whereas those with levels below $200 \mathrm{mg} / \mathrm{dl}$ were considered free of diabetes. The STZ/TcS-2-treated group showed a lower percentage of T1D incidence than the STZ and STZ/TcS-1 groups. (d) Glucose tolerance test for all groups. ${ }^{*}$ Differences between STZ versus untreated group. ${ }^{*}$ Differences between STZ/TcS-1 versus untreated group. ${ }^{\$}$ Differences between STZ/TcS-2 versus Untreated group. The data represent at least 3 independent experiments; $N=5$ mice per group. For comparisons between two groups, the Mann-Whitney $U$ test was applied to test differences with nonparametric data. Experiments with multiple groups were tested by the Kruskal-Wallis test followed by Dunn's multiple comparison test. Mean \pm SEM. ${ }^{*} p<0.05,{ }^{* *} p<0.01$, and ${ }^{* * *} p<0.001$. 

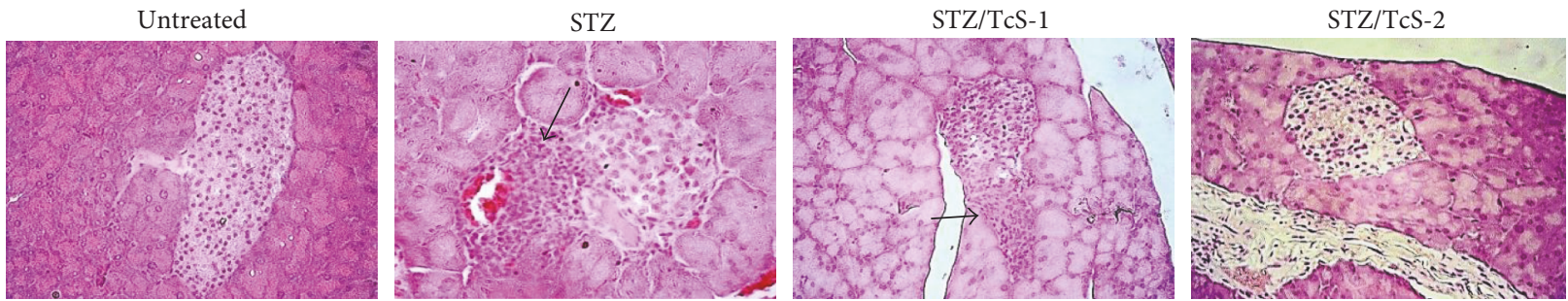

(a)

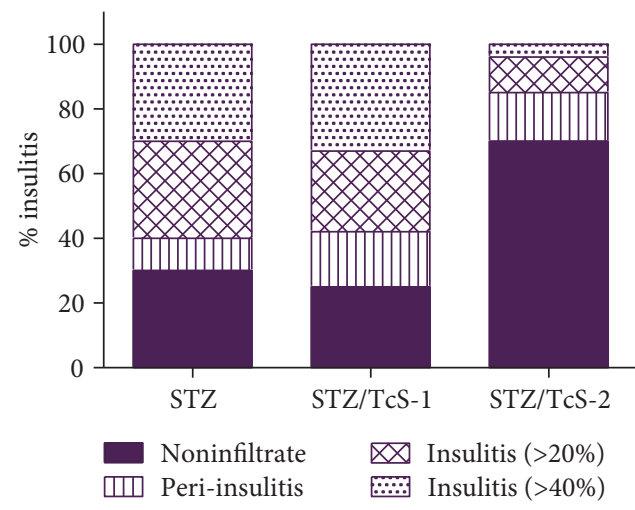

(b)

Figure 2: Constant TcS exposure reduces insulitis in MLD-STZ-treated mice. T1D-induced mice were treated with TcS for 6 weeks. The animals were sacrificed, and the pancreases were processed, embedded in paraffin, and cut into $5 \mu \mathrm{m}$ sections. (a) Representative pictures of islets of Langerhans stained with $\mathrm{H} \& \mathrm{E}$ and evaluated microscopically for the presence of insulitis. Arrows show cellular infiltrate in islets of Langerhans. Magnification of 400x. (b) Percentage of insulitis. Score of infiltrated islets. Noninfiltrated: without damage. Periinsulitis: infiltration only at the periphery of the islets. Insulitis $20 \%$ : infiltration of $\geq 20 \%$ or more but less than $40 \%$. Insulitis $40 \%$ : infiltrate of $\geq 40 \%$ of islets. The insulitis evaluation shown is representative of 10 mice per group (at least 100 islets were counted).

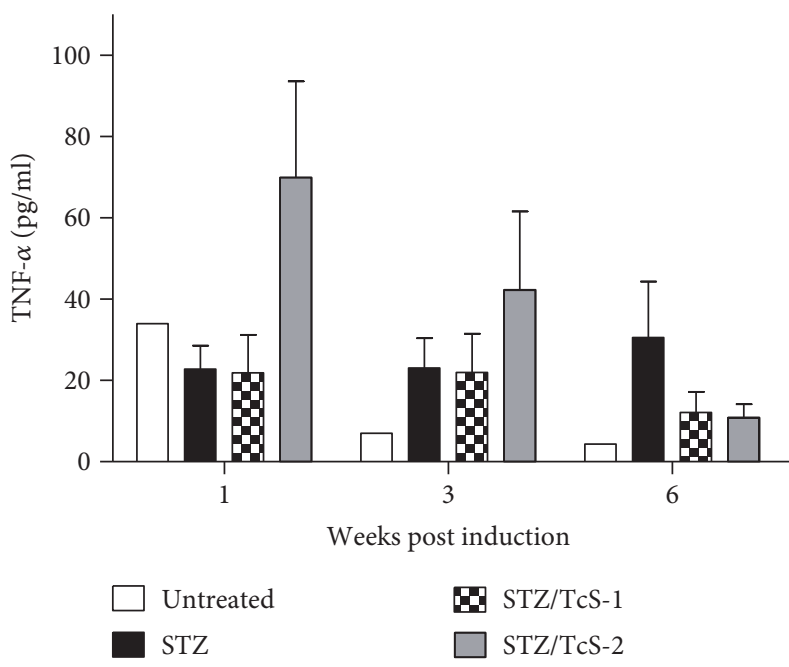

(a)

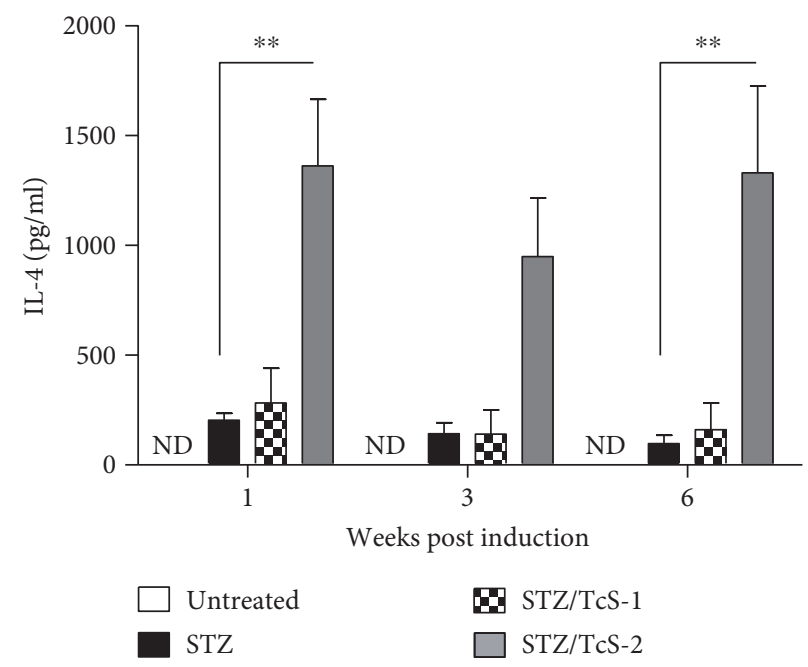

(b)

FIgURE 3: Injection of T. crassiceps soluble antigen increases IL-4 in serum. Serum was collected from mice at 1, 3, and 6 weeks p.i. (a) TNF- $\alpha$ and (b) IL- 4 were detected by ELISA sandwich. ND $=$ nondetected. ${ }^{* *} p<0.01$ by the Mann-Whitney $U$ test. Data are representative of two independent experiments. $N=4$ mice per group.

3.7. Macrophage Depletion Reverses Helminth-Associated Protection against T1D. To elucidate which cell population was participating in helminth-associated protection against

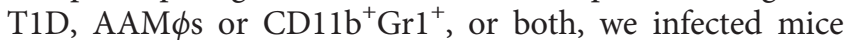
with T. crassiceps metacestodes, and 6 weeks later, T1D was induced in the infected mice with MLD-STZ. Later, in the second week post T1D induction, diabetic mice were injected i.p. 3 times a week with $2 \mathrm{mg} /$ mouse of clodronate-loaded liposomes or control PBS-loaded liposomes for 6 weeks post T1D induction (Figure 7(a)). Diabetic mice treated with PBS- 

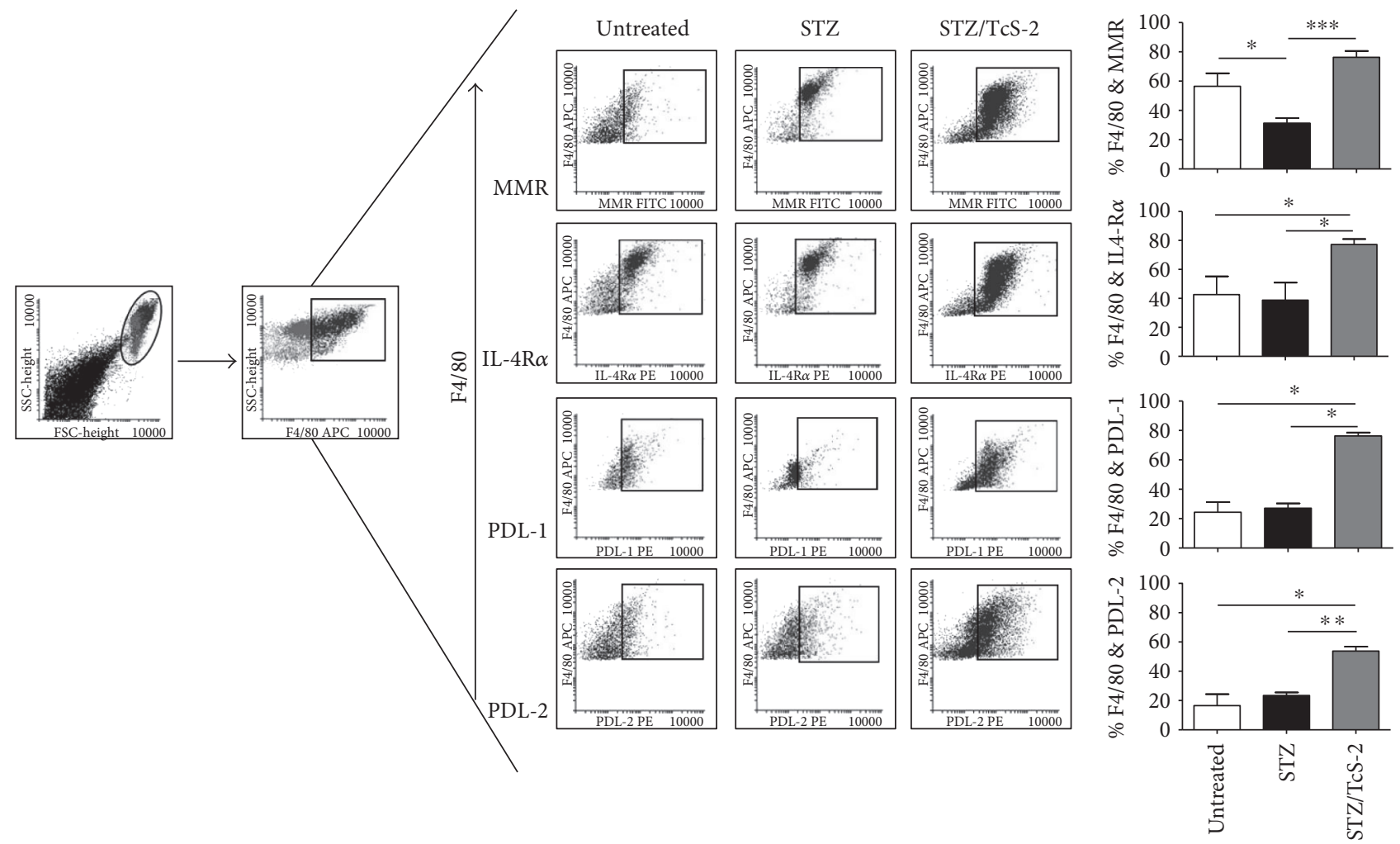

Figure 4: AAM $\phi$ s appear with constant soluble antigen treatment. PECs were obtained at 6 weeks post induction and stained for AAM $\phi$ markers such as MMR, IL-4R $\alpha$, PDL-1, and PDL-2. Its expression was evaluated in F4/80-positive cells by flow cytometry. The data represent at least 2 independent experiments. The data shown are the mean \pm SEM. ${ }^{*} p<0.05,{ }^{* *} p<0.01$ by the Mann-Whitney $U$ test, and ${ }^{* * *} p<0.001 . N=4$ mice per group.

loaded liposomes (STZ PBS) and clodronate-loaded liposomes (STZ Cl) displayed hyperglycemia beginning in the second week post-T1D induction. In contrast, T. crassicepsinfected mice receiving control liposomes (STZ/Tc PBS) displayed levels of blood glucose below $200 \mathrm{mg} / \mathrm{dl}$ during whole treatment (Figure 7(b)). Interestingly, when T. crassiceps-infected mice received clodronate-loaded liposomes (STZ/Tc $\mathrm{Cl}$ ), the protective effect of this helminth infection was reversed, and mice turned as diabetic as STZ mice, showing hyperglycemia (Figure 7(b)). The incidence of diabetes was also critically affected by clodronate treatment in each group: all STZ PBS mice displayed hyperglycemia by the second week post T1D induction and were considered diabetic, while the STZ Cl mice showed hyperglycemia until the fourth week post T1D induction. In contrast, in the STZ/Tc PBS group, a protective effect was observed, only $50 \%$ of these mice had hyperglycemia $(>200 \mathrm{mg} / \mathrm{dl})$, and the rest of the animals remained healthy, but when T. crassiceps-infected animals received clodronate liposomes (STZ/Tc Cl), all these animals rapidly turned diabetic, with high levels of glucose at the sixth week, and only $15 \%$ were free of diabetes (Figure $7(\mathrm{c})$ ). Finally, to gain further insight about whether $\mathrm{AAM} \phi \mathrm{s}, \mathrm{CD} 11 \mathrm{~b}^{+} \mathrm{Gr} 1^{+}$cells, or both were involved in the protection of diabetic mice and whether clodronateloaded liposomes depleted any other regulatory cell, flow cytometry of peritoneal cells was performed. We observed that STZ mice receiving PBS-loaded liposomes recruited
$\mathrm{FSC}^{\text {high }} \mathrm{SSC}^{\text {high }} \mathrm{F} 4 / 80^{+}$and $\mathrm{FSC}^{\text {high }} \mathrm{SSC}^{\text {high }} \mathrm{CD} 11 \mathrm{~b}^{+}$cells in the peritoneal cavity; in contrast, mice receiving clodronateloaded liposomes displayed a depleted $\mathrm{F} 4 / 80^{+}$cell population but maintained the recruitment of $\mathrm{CD} 11 \mathrm{~b}^{+}$cells, demonstrating that clodronate liposomes only depleted macrophages, mostly $\operatorname{AAM} \phi$ s, and no other populations such as $\mathrm{CD} 11 \mathrm{~b}^{+}$ cells (Figure 7(d)). Figure 7(e) shows that $T$. crassiceps infection in conjunction with STZ significantly increased the markers MMR and PDL-2 compared with all other groups, whereas infected mice that received clodronate liposomes showed a significant reduction in the expression of these $\operatorname{AAM} \phi$ markers.

Next, we look for any differences between two different subpopulations of MDSCs, CD $11 b^{+} \mathrm{Ly}_{6 \mathrm{C}^{+}} \mathrm{Ly} 6 \mathrm{G}^{-}$and

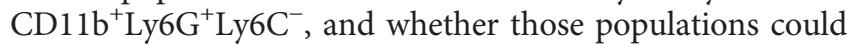
change their recruitment when we injected clodronateloaded liposomes in diabetic animals. Flow cytometry of peritoneal cells shows that all groups expressed $\mathrm{CD} 11 \mathrm{~b}^{+} \mathrm{Ly}_{6 \mathrm{C}}{ }^{+}$ markers at a high percentage except for the untreated group. However, when animals received clodronate liposomes, the percentage of $\mathrm{CD} 11 \mathrm{~b}^{+} \mathrm{Ly} 6 \mathrm{C}^{\text {high }}$ cells was significantly higher than in mice receiving PBS liposomes (Figure 7(f)). In contrast, granulocytic cells, CD $11 \mathrm{~b}^{+} \mathrm{Ly} 6 \mathrm{C}^{-} \mathrm{Ly} 6 \mathrm{G}^{+}$, increased with the injection of clodronate loaded-liposomes; STZ/Tc $\mathrm{Cl}$ and STZ/Tc PBS had significant differences compared to mice from the STZ PBS group. Both the STZ/Tc Cl and STZ $\mathrm{Cl}$ groups displayed higher percentages of both 


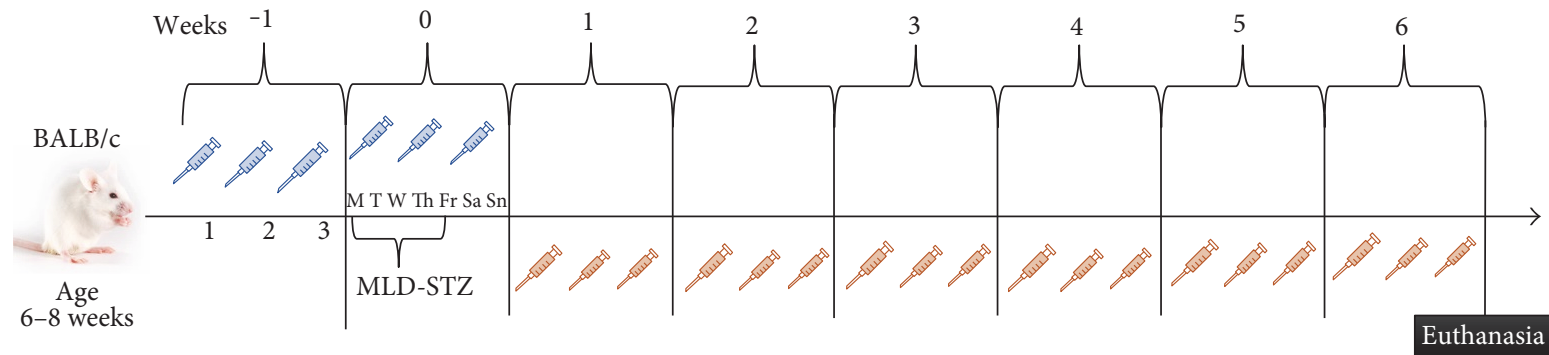

Injection of TcES-1

Injection of TcS-3, TcS-4,

and TcS-5 or injection of

TcES-2, TcES-3, and TcES-4

(a)

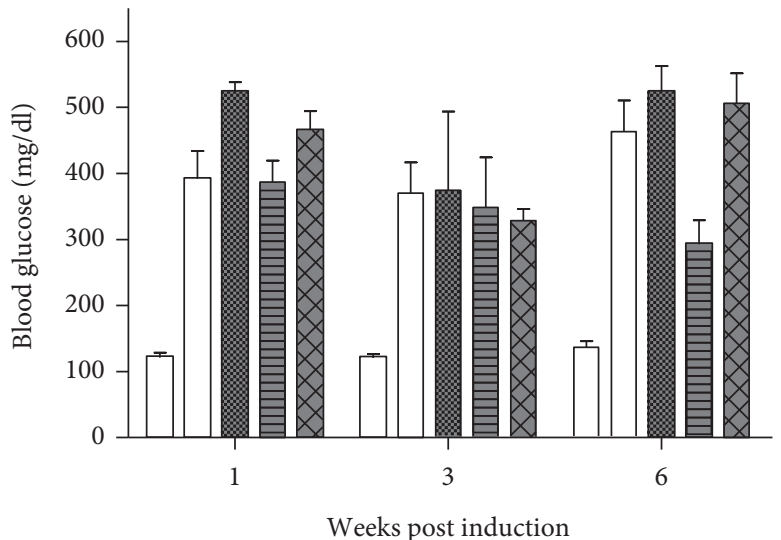

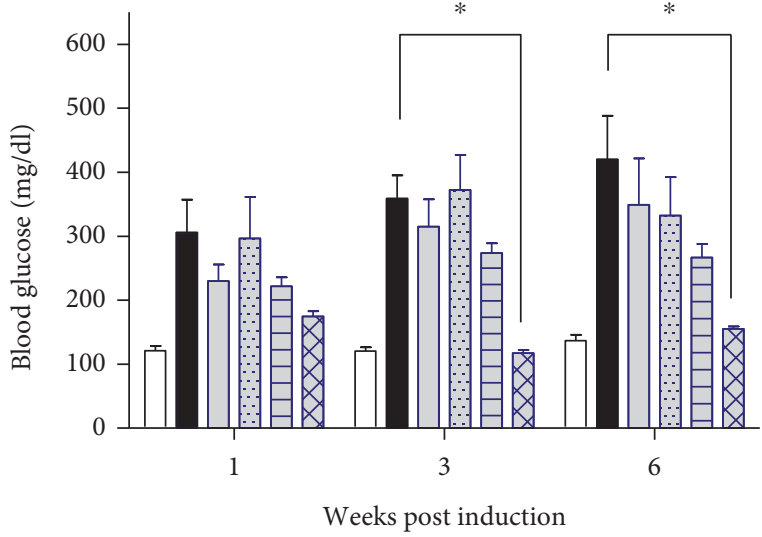

$\square$ Untreated

STZ

STZ/TCES-1

(c)
… STZ/TcES-2

STZ/TcES-3

XTZ/TcES-4

(b)

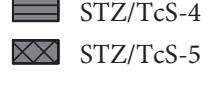

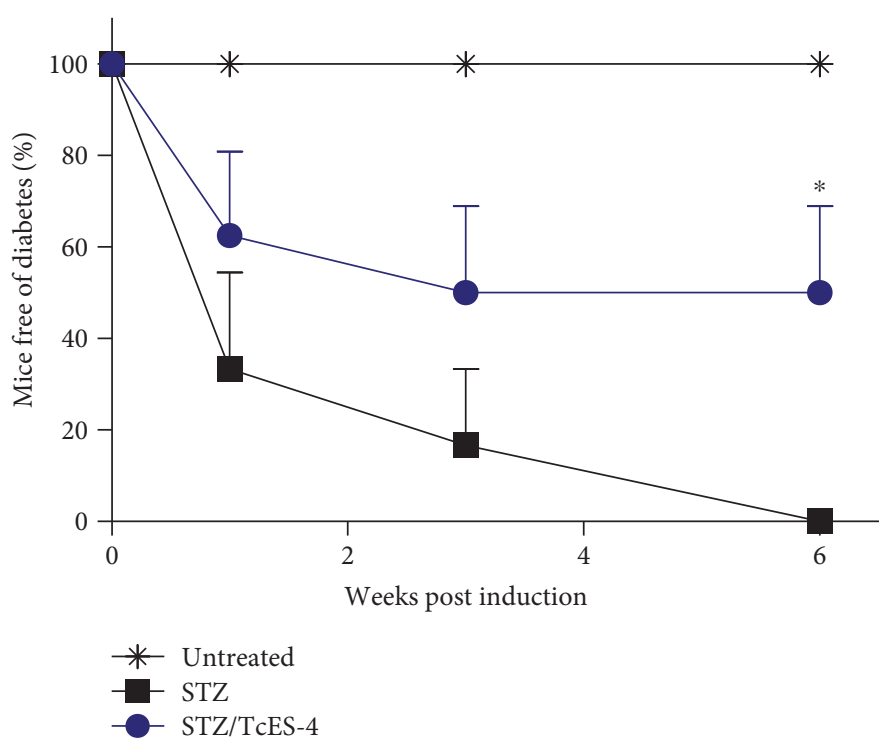

(d)

Figure 5: Continued. 

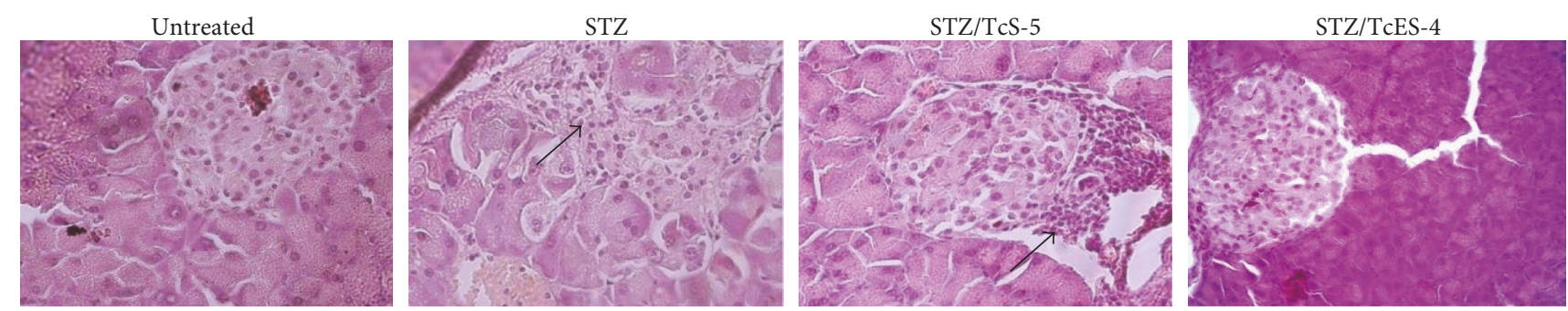

(e)

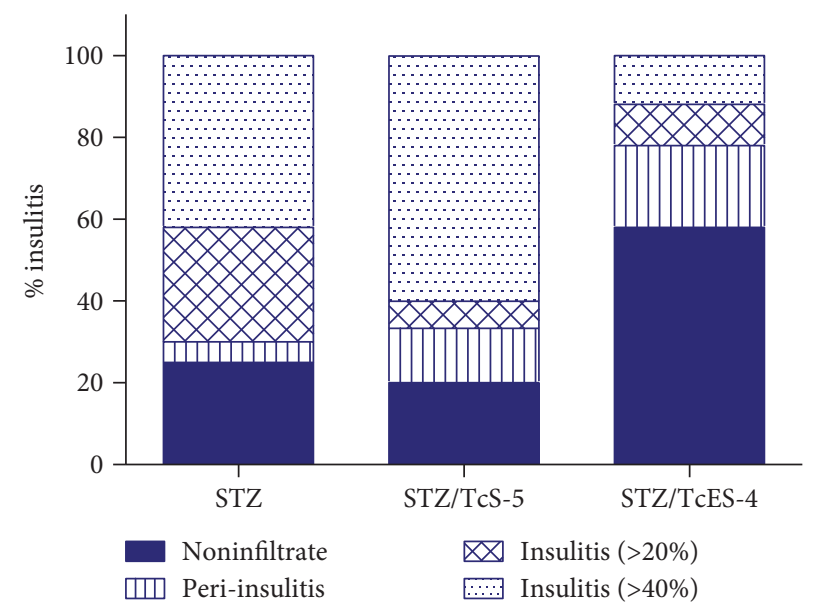

(f)

Figure 5: Exposure to TcES reduces T1D damage. Mice were injected with soluble antigen (TcS) or excreted/secreted antigen (TcES). Blood glucose levels were measured for 6 weeks after T1D induction. (a) Methodology diagram illustrating injection of T. crassiceps antigen (TcS and TcES). (b) Blood glucose of mice treated with different doses of TcS post T1D induction. (c) Glycemia of mice receiving constant and post T1D induction treatment with TcES. (d) Percentage of mice free of diabetes. Mice with glycemia greater than $200 \mathrm{mg} / \mathrm{dl}$ were considered diabetic, whereas those with levels of $200 \mathrm{mg} / \mathrm{dl}$ or lower were considered free of diabetes. ${ }^{*}$ Differences between STZ and STZ/TcES-4. (e) Histology of pancreas. Arrows represent insulitis and pancreatic islet damage. (f) Insulitis score. Noninfiltrated: without damage. Peri-insulitis: infiltration only at the periphery of the islets. Insulitis $20 \%$ : infiltration of $\geq 20 \%$ or more but less of $40 \%$. Insulitis $40 \%$ : infiltration of $\geq 40 \%$ of islets. Data are representative of 2 independent experiments. $N=5$ mice per group. For comparisons between two groups, the Mann-Whitney $U$ test was applied to test differences with nonparametric data. Experiments with multiple groups were tested by the Kruskal-Wallis test followed by Dunn's multiple comparison test. The data shown are the mean \pm SEM. ${ }^{*} p<0.05$.

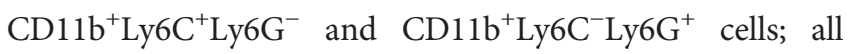
these observations suggest that monocytic and/or granulocytic MDSCs could promote an inflammatory response and damage because both groups showed hyperglycemia and incidence of T1D; and furthermore, the recruitment of $\operatorname{AAM} \phi s$ was less evident in these groups. In contrast, STZ/ Tc PBS mice maintained AAM $\phi$ recruitment and displayed both reduced glycemia and reduced incidence of T1D, suggesting that $\mathrm{AAM} \phi$ s were regulatory cells with an important role in modulating T1D induced by MLD-STZ.

This last experimental design was replicated with animals exposed to TcS, where mice were injected with TcS one week before T1D induction and for 4 weeks afterward. Data shown in Figure 8 indicate that mice exposed to TcS and treated with PBS liposomes (STZ/TcS PBS) displayed reduced hyperglycemia, whereas mice similarly exposed to TcS but receiving clodronate-loaded liposomes (STZ/TcS $\mathrm{Cl}$ ) are not protected against increasing blood glucose levels, again supporting the idea that $\mathrm{AAM} \phi$ s may play a central role in T1D protection using helminth-derived molecules.

\section{Discussion}

Helminths and their products have been suggested as a powerful weapon against many inflammatory diseases, such as type 1 diabetes, arthritis, colitis, encephalomyelitis, Crohn's disease, and asthma [8,32-34]; such effects are mainly based on the ability of helminths and their antigens to induce strong Th2-biased responses, with increases in cytokines such as IL-4, IL-10, IL-13, and TGF- $\beta[19,35]$. Additionally, they induce regulatory cells, such as $\operatorname{AAM} \phi s$, Treg, and MDSCs, which have been linked with decreased inflammatory responses and less tissue damage as well as with wound healing processes [18, 27, 36, 37].

Type 1 diabetes is an autoimmune disease in which insulin-producing $\beta$-cells are destroyed by $\mathrm{CD} 4^{+}$and $\mathrm{CD} 8^{+}$ $\mathrm{T}$ cells and CAM $\phi$ s $[1,2]$. Studies in animal models such as NOD or MLD-STZ-induced T1D mice have provided evidence for the ability of helminths to reduce inflammatory responses, death of $\beta$-cells, and insulitis [7]. In a previous study, we showed that $T$. crassiceps infection was able to 

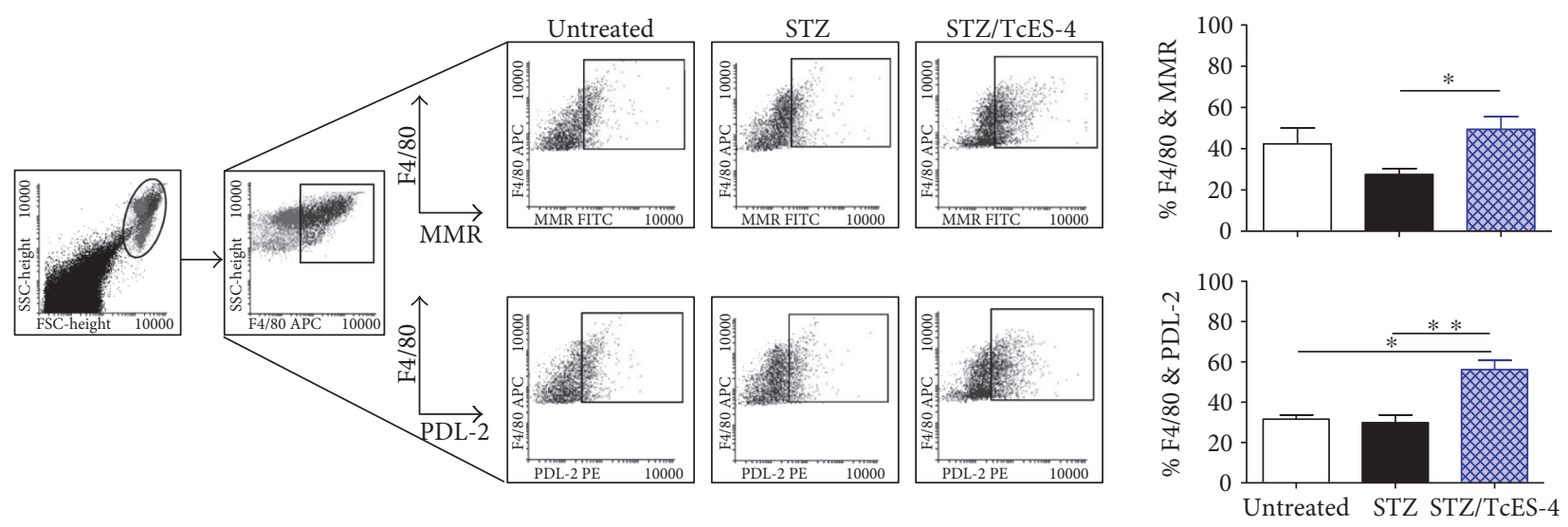

(a)

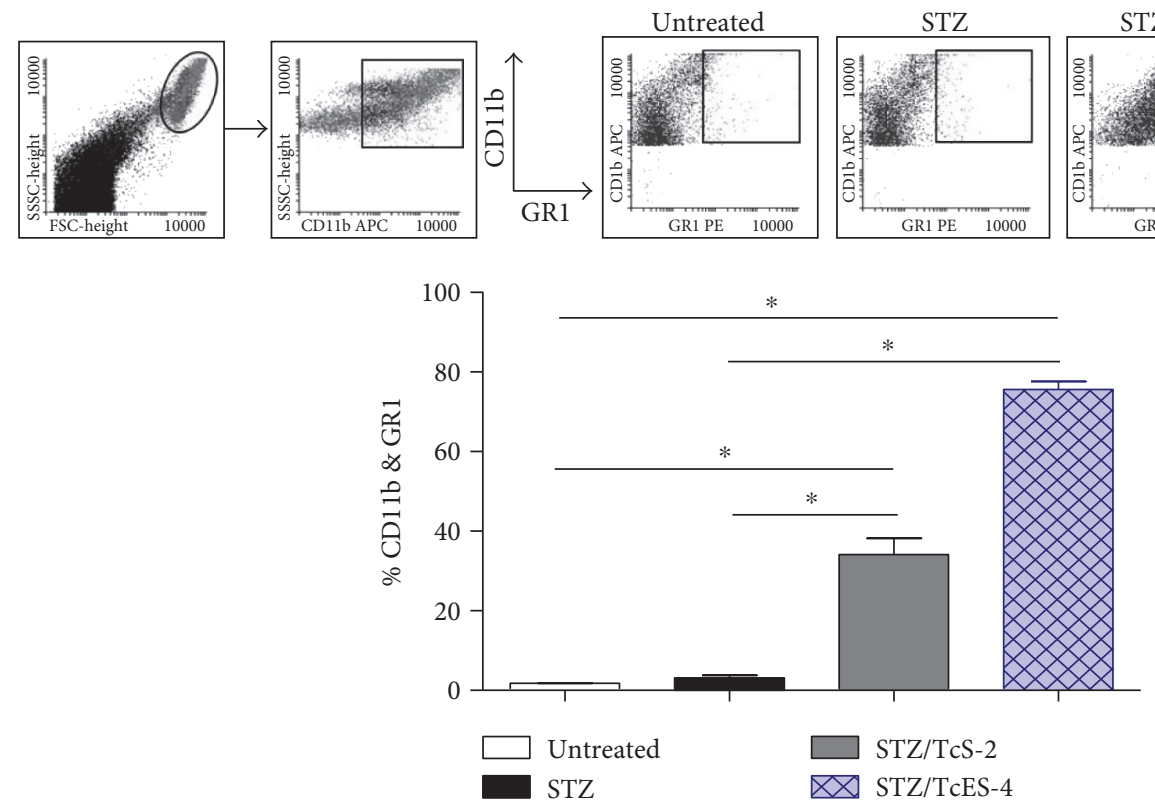

(b)

FIgure 6: Exposure to TcES during T1D increases both AAM $\phi$ and FSC ${ }^{\text {high }} \mathrm{CD} 11 \mathrm{~b}^{+} \mathrm{Gr}^{+}$populations. (a) PECs were stained for F4/80, MMR, and PDL2 and analyzed in a flow cytometer. (b) PECs were stained for the markers Gr1, CD11c, and CD11b and analyzed by flow cytometry. ${ }^{*} p<0.05$ by the Mann-Whitney $U$ test. ${ }^{* *} p<0.01$. Data are representative of 2 independent experiments. $N=4$ mice per group.

reduce hyperglycemia and insulitis associated with strong recruitment of $\mathrm{AAM} \phi s$; such data suggest that these cells could be important to prevent pancreatic damage and suppress autoreactive $\mathrm{T}$ cells [13]. Additionally, our group showed that T. crassiceps infection could decrease inflammatory response and damage by autoreactive cells in colitis and experimental autoimmune encephalomyelitis (EAE) [38, 39]. Our goal in the present work was to investigate whether T. crassiceps products (TcES or TcS) would mimic the effect of experimental infection by decreasing inflammatory responses induced by T1D, and we looked for cells involved in this regulatory response. Here, we demonstrate that both treatments, TcS and TcES, can regulate hyperglycemia, insulitis, and the incidence of T1D, but with slight differences. We found that it is important for TcS treatment to begin before MLD-STZ induction of T1D and continue with constant injections of TcS for a protective effect. Otherwise, TcES treatment in diabetic mice was more effective to decrease hyperglycemia at a high dose $(200 \mu \mathrm{g})$, compared with TcS $(50 \mu \mathrm{g})$, in a post induction system of MLD-STZ-induced T1D. Differences between the effects of TcS and TcES may be explained by a previous work in which exposure of macrophages to TcES modified their inflammatory response to IFN- $\gamma$ through the expression of high levels of SHP-1 and SOCS3, which are suppressors of IFN- $\gamma$-transducing signaling, but when macrophages were exposed to TcS, these cells did not block IFN- $\gamma$ signaling [40]. In another research, it was found that $T$. crassiceps E/S antigen induced a tolerogenic phenotype in dendritic cells, stopped their maturation, and downmodulated the expression of costimulatory molecules [29]. Such differences observed in vivo and in vitro between TcS and TcES may be associated with differences in the composition or concentration of biomolecules found in the $>50 \mathrm{kDa}$ fraction. 


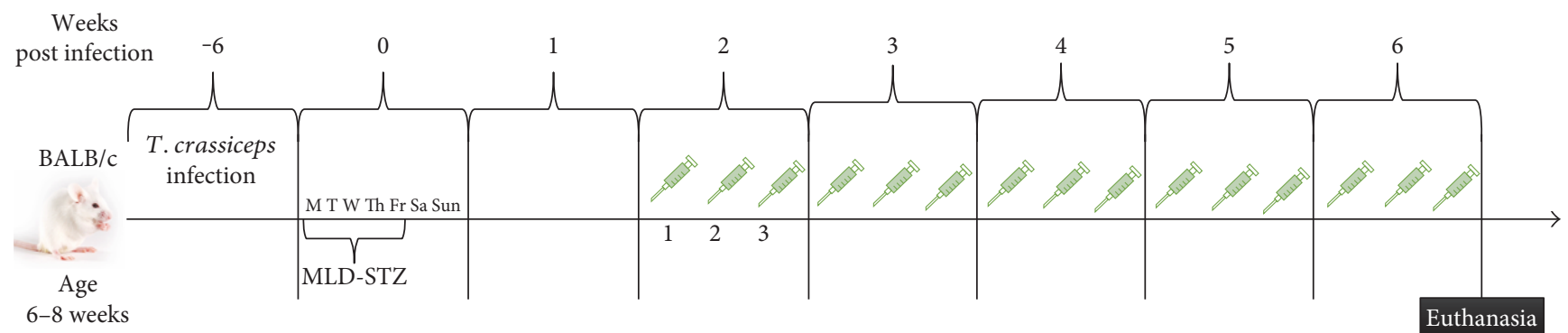

Injection of clodronate- or PBS-loaded liposomes

(a)

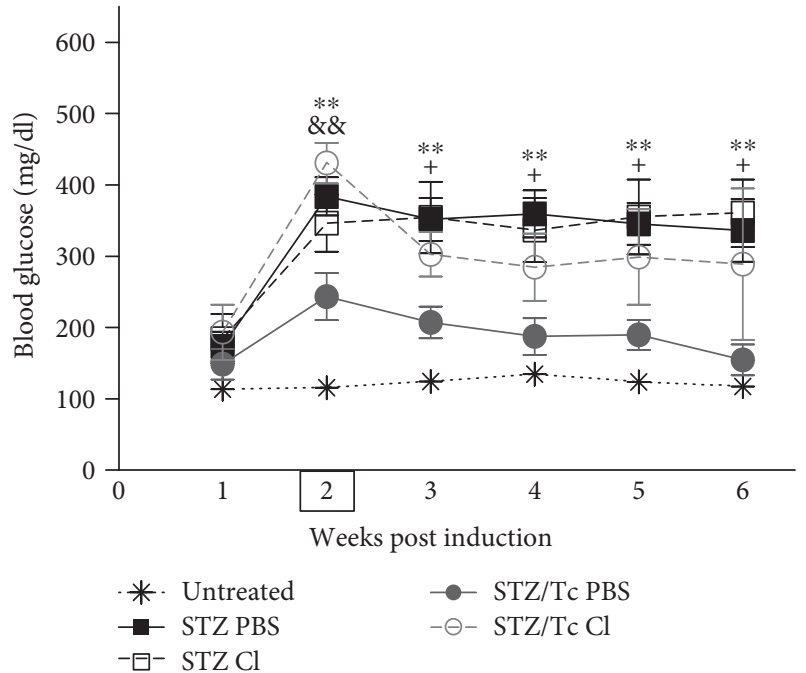

(b)

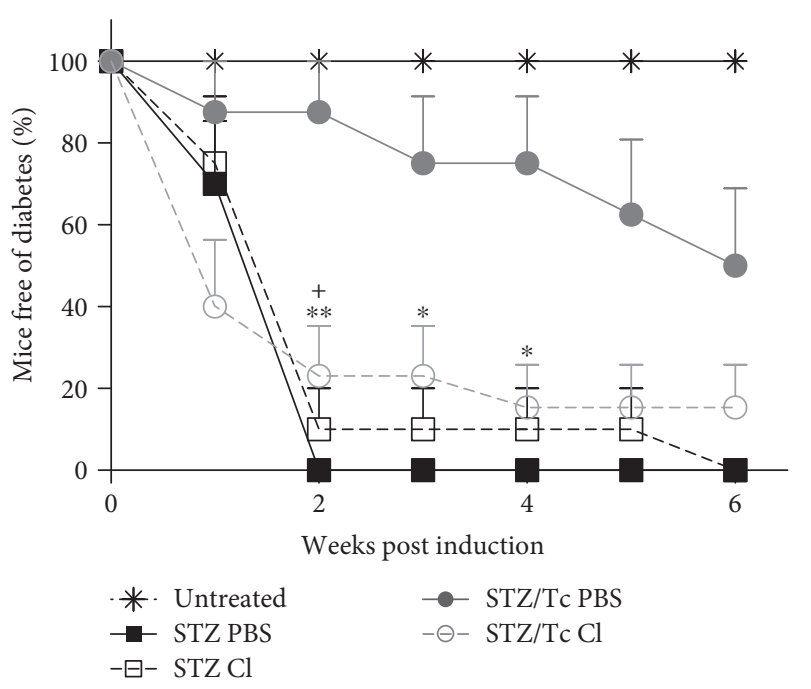

(c)
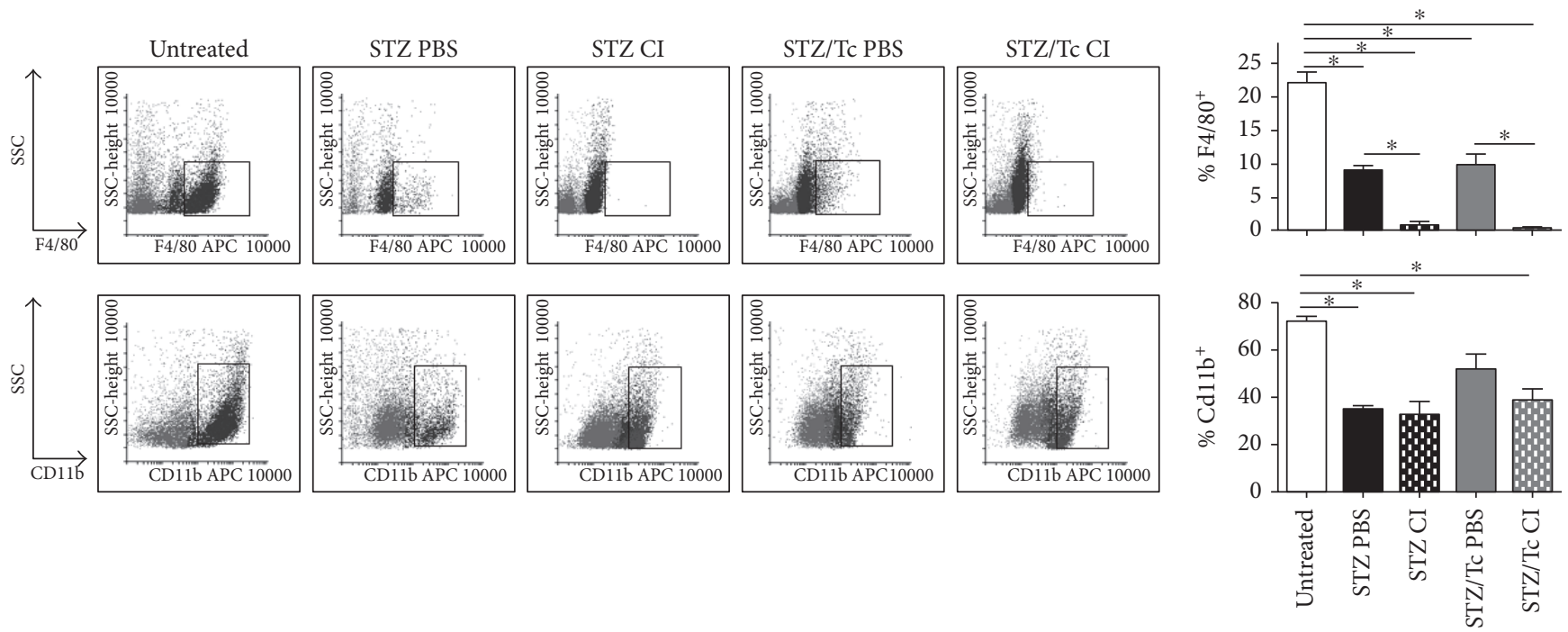

(d)

Figure 7: Continued. 

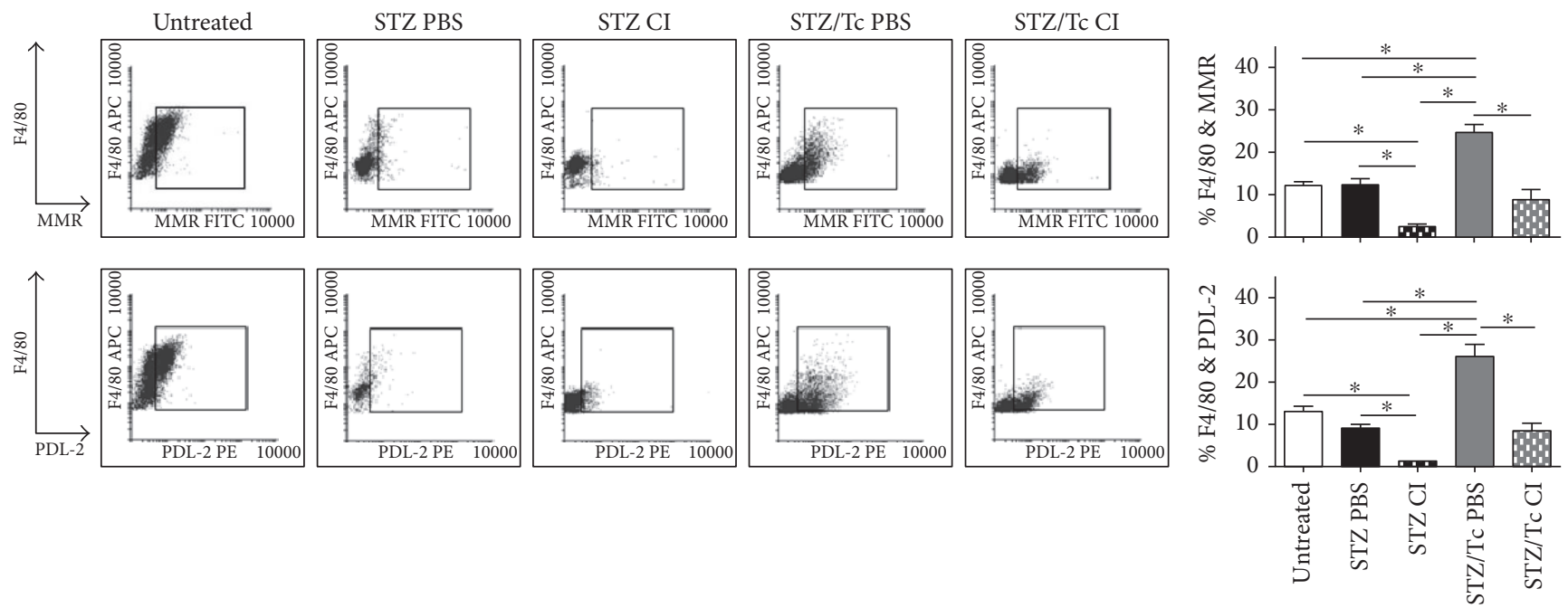

(e)
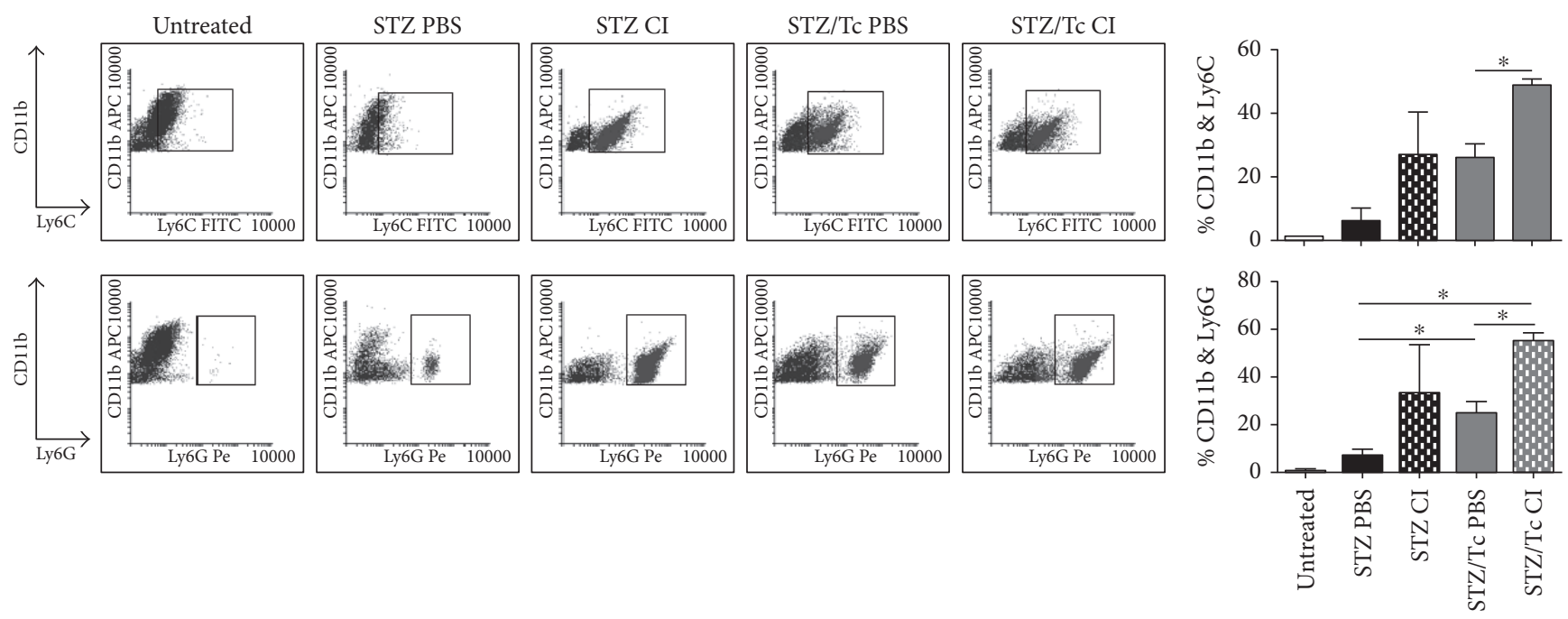

(f)

FIGURE 7: Clodronate treatment reveals a role for AAM $\phi$ s in T1D protection. Mice were infected i.p. by T. crassiceps metacestodes. After the sixth week post infection, mice were induced by MLD-STZ and then were injected with clodronate-loaded liposomes or PBS-loaded liposomes, 3 times for a week during four weeks p.i. T1D. (a) Diagram of experimental design. (b) Blood glucose of mice during 6 weeks post T1D induction. ( ${ }^{*}$ Differences between STZ/Tc PBS and STZ PBS. ${ }^{+}$Differences between the STZ/Tc PBS group and STZ Cl. ${ }^{\& \&}$ Differences between STZ/Tc PBS and STZ/Tc Cl). (c) Mice free of diabetes. Percent of mice free of diabetes (glycemia higher than $200 \mathrm{mg} / \mathrm{dl}$ were considered diabetic). ${ }^{*}$ Differences between STZ and STZ/Tc PBS. (d) Flow cytometry of FSC ${ }^{\text {high }} \mathrm{F} 4 / 80^{+}$and FSC ${ }^{\text {high }} \mathrm{CD} 11 \mathrm{~b}^{+}$peritoneal cells. (e) Flow cytometry of PECs marked for F4/80, MMR, and PDL-2. (f) CD11b ${ }^{+}$Ly6C ${ }^{+}$and $\mathrm{CD} 11 \mathrm{bLy} 6 \mathrm{G}^{+}$ cells were analyzed in a FACs. For comparisons between two groups, the Mann-Whitney $U$ test was applied to test differences with nonparametric data. Experiments with multiple groups were tested by the Kruskal-Wallis test followed by Dunn's multiple comparison test. The data shown are the mean \pm SEM. ${ }^{*} p<0.05$ and ${ }^{* *} p<0.01$. Data are representative of 2 independent experiments. $N=4$ mice per group.

Different studies have demonstrated the ability of several helminth-derived products to reduce T1D development; however, most of them were evaluated as a pretreatment for T1D. For example, Zaccone et al. showed that S. mansoni products may prevent the development of diabetes in NOD mice, but only if the treatment was started before the fourth week of age [21]. In other research, Amdare et al. showed that treatment of B. malayi ES and soluble (adult and microfilaria) product may protect against T1D, but only if the series of injections were started before T1D induction [41]. More recently, in 2016, Ajendra et al. demonstrated that
L. sigmodontis antigen (crude worm extract) may protect against T1D in later treatments (after 10 weeks of age) in NOD mice, but only as part of a combined therapy with an intranasal proinsulin dosage [42]. In this context, we believe that TcES could be a promising treatment, considering that its effects do not require additional therapy for protection against T1D. Furthermore, with the reasoning that the diagnosis of T1D in humans is reached late, when most of the $\beta$-cells are destroyed, TcES may prevent or delay pancreatic islet destruction after T1D initiation. In line with this idea, another important question is knowing if T. crassiceps 


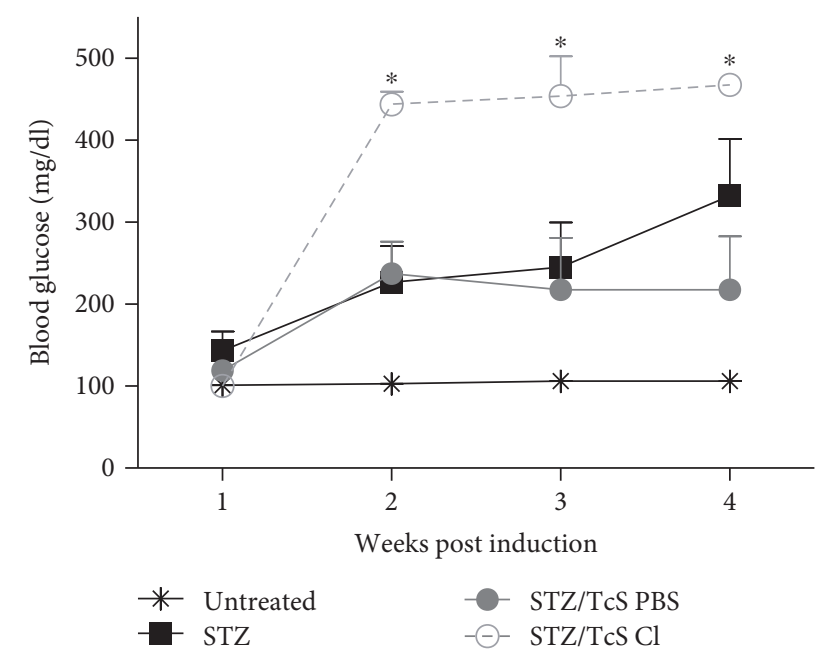

FIGURE 8: Clodronate treatment abrogates protection against $\mathrm{T} 1 \mathrm{D}$ in mice receiving TcS. The clodronate-liposome injections followed the same experimental design as the $T$. crassiceps infection process. Mice were injected with TcS 1 week before T1D induction and continue receiving the treatment for 4 weeks post T1D induction. Blood glucose was measured for 4 consecutive weeks. ${ }^{*}$ Differences between STZ/TcS Cl and STZ/TcS PBS. Data are representative by 2 independent experiments, each group with 4 mice. Mean \pm SEM. ${ }^{*} p<0.05$ by the Mann-Whitney $U$ test.

antigens can be recognized by human cells; in this regard, Terrazas et.al. showed that human dendritic cells exposed to $T$. crassiceps excreted/secreted antigens displayed a tolerogenic profile [29]. Further studies are needed to prove that TcES may regulate human autoreactive cells as a treatment in T1D in the future.

Another important point about the effect of helminths and their products is their capability to recruit regulatory cells involved in the downregulation of inflammation, as has been demonstrated by several helminth infections ( $S$. mansoni, $H$. polygyrus, B. malayi, N. brasiliensis, T. spiralis, $F$. hepatica, and T. crassiceps) reducing T1D development; however, all of them were also evaluated as a pretreatment for T1D, and most of them recruited $\operatorname{AAM} \phi s[8,9,11,12]$. Some research has also been focused on products of helminths to search for regulatory cells involved in downregulating inflammation. For example, S. mansoni products (SEA and SWA) could induce high numbers of $\mathrm{AAM} \phi \mathrm{s}$ and Treg cells $[20,21]$. E/S products of $F$. hepatica also increased AAM $\phi$ and Treg populations in diabetic mice, suggesting that both populations are related to protection against diabetes [23]. In our previous research, we found that T. crassiceps infection in MLD-STZ-induced T1D mice recruited AAM $\phi s$ [13]. Here, we gain knowledge on the role of $\mathrm{AAM} \phi$ s during T. crassiceps antigen treatment (TcS and TcES) in T1D, where we find increased expression of $\mathrm{AAM} \phi$ markers such as MMR, PDL-1, and PDL-2 in T1Dprotected mice. These data agree with the recent observation that reduction of hyperglycemia in mice infected with $S$. mansoni was Treg independent, while recruitment of $\operatorname{AAM} \phi$ s was important to the process [43]. In that work, the authors blocked Treg cells by injecting a specific antiTreg antibody, and hyperglycemia did not improve or even turned worse. However, these same authors did not test a specific role for $\operatorname{AAM} \phi s$, whereas in this investigation we show for the first time that $\mathrm{AAM} \phi$ s are critical for the protective effect of helminth-derived products in T1D development. Moreover, whereas in the $S$. mansoni infection there was only a limited reduction of hyperglycemia and all the animals became diabetic after 3 weeks, here we showed that T. crassiceps-derived molecules or even the whole infection was able to reduce the incidence of T1D by $50 \%$ for at least 6 weeks. Together, these data indicate that T. crassiceps and their derived molecules display more potent antiinflammatory activity than that observed in other helminthic infections and that such protective effect is highly dependent on the presence of $\mathrm{AAM} \phi \mathrm{s}$.

Additionally, we observed that TcS and TcES exposure directly upregulated the recruitment of $\mathrm{CD}_{11} \mathrm{~b}^{+} \mathrm{Gr} 1^{+}$cells in diabetic mice compared with STZ and untreated mice. MDSCs have garnered increased interest because they can suppress $\mathrm{T}$ cell responses. In 2010, Yin et al. [44] demonstrated that adoptive transference of MDSCs in NOD mice significantly decrease diabetes onset as well as pancreatic islet damage, and these authors suggested that MDSCs might mediate anergy of autoreactive $\mathrm{T}$ cells and favor the presence of Treg cells. Conversely, in other research, MDSCs were associated with damage, because these cells were favored differentiation of $\mathrm{CD} 4^{+}$cells towards a Th17 profile in the pathogenesis of EAE in mice [45].

To elucidate which cells may have a more significant role downregulating T1D in our system, we decide to deplete phagocytic cells by i.p. injections of clodronate-loaded liposomes during both T. crassiceps infection and TcS exposure. Previously, it has been demonstrated that clodronateloaded liposomes only depleted phagocytic cells (mostly macrophages) in our system of peritoneal injections, without affecting dendritic cells and eosinophils [31]. Here, we found that $\mathrm{AAM} \phi$ s were significantly reduced in STZ/Tc $\mathrm{Cl}$ mice, while STZ/Tc PBS recruited higher percentages of AAM $\phi$ s. However, we also found that $\mathrm{CD}_{11} \mathrm{~b}^{+} \mathrm{Ly} 6 \mathrm{C}^{+} \mathrm{Ly} 6 \mathrm{G}^{-}$and $\mathrm{CD} 11 \mathrm{~b}^{+} \mathrm{Ly} 6 \mathrm{C}^{-} \mathrm{Ly}_{6} \mathrm{G}^{+}$populations were increased in mice receiving clodronate liposomes (STZ Cl and STZ/Tc Cl), with significant differences from the other groups. Recently, our group demonstrated that high recruitment of CD11b ${ }^{+}$Ly6$\mathrm{C}^{\text {high }}$ in colitic mice was associated with damage and colon inflammation [38]. In line with this previous report, the present study found a similar outcome in T1D mice, with an elevated number of MDSCs (monocytic and granulocytic) in the peritoneal cavity of mice receiving clodronate, but without protection against T1D development; thus, our data strongly suggest that granulocytic and monocytic cells do not participate as a protective cell population in our system. Further studies are needed to elucidate the role of MDSCs in diabetic mice, such as to demonstrate that these cells have suppressive ability, a key feature to name these cells. Instead, a strong role for AAM $\phi$ s in the anti-T1D effects of Taenia products can be assumed; given that $\mathrm{STZ} / \mathrm{Tc} \mathrm{Cl}$ reversed the protective effect of $T$. crassiceps infection, producing similar hyperglycemia levels to STZ and STZ Cl mice and T1D incidence did reach up to $100 \%$, while the STZ/Tc PBS group was protected up to $50 \%$, displaying hyperglycemia levels 
under $200 \mathrm{mg} / \mathrm{dl}$. A reversal of the protective effect of TcS was also observed when mice exposed to $\mathrm{TcS}$ received clodronate liposomes. However, clodronate may also affect CAM $\phi$ s and thus eliminate a dangerous cell population for T1D development, but given the worsening of the glucose levels, this possible fact may support a more critical role for $\mathrm{CD}^{+}$and $\mathrm{CD}^{+}$autoimmune cells in tissue damage. Together, these results strongly support the hypothesis that $\mathrm{AAM} \phi \mathrm{s}$ recruited by $T$. crassiceps infection and $T$. crassiceps-derived products are a key population that importantly reduces inflammatory responses associated with T1D development.

\section{Conclusion}

In conclusion, our findings indicate that exposure to TcS and TcES has a potential protective effect against MLD-STZinduced T1D development by reducing hyperglycemia and the incidence of this autoimmune disease. Mechanistically, our new data support the hypothesis that $\mathrm{AAM} \phi$ s recruited by T. crassiceps-derived products play a critical role in downregulating $\mathrm{T} 1 \mathrm{D}$, because when this population is depleted early, the protective effect is abrogated. It is necessary to elucidate the putative signaling pathways triggered by these $T$. crassiceps-derived products to understand more completely the mechanisms associated with their anti-inflammatory and antidiabetic effects.

\section{Conflicts of Interest}

The authors declare that there is no conflict of interest regarding the publication of this paper.

\section{Acknowledgments}

This work was supported by CONACYT (Grants 167799 and 280013) and by Instituto de Ciencia y Tecnología del Distrito Federal (319-2010 and PINVII-16) (301/2011). It is part of the requirements to obtain the $\mathrm{PhD}$ degree in the Programa de Doctorado en Ciencias Biomédicas, Universidad Nacional Autónoma de México, of Arlett Espinoza-Jiménez, who was supported by a CONACYT fellowship (215929), México.

\section{References}

[1] M. M. Jahromi and G. S. Eisenbarth, "Cellular and molecular pathogenesis of type 1A diabetes," Cellular and Molecular Life Sciences, vol. 64, pp. 865-872, 2007.

[2] M. C. Denis, U. Mahmood, C. Benoist, D. Mathis, and R. Weissleder, "Imaging inflammation of the pancreatic islets in type 1 diabetes," Proceedings of the National Academy of Sciences of the United States of America, vol. 101, no. 34, pp. 12634-12639, 2004.

[3] A. Rabinovitch and W. L. Suarez-Pinzon, "Role of cytokines in the pathogenesis of autoimmune diabetes mellitus," Reviews in Endocrine and Metabolic Disorders, vol. 4, no. 3, pp. 291-299, 2003.

[4] M. Cnop, N. Welsh, J. C. Jonas, A. Jörns, S. Lenzen, and D. L. Eizirik, "Mechanisms of pancreatic $\beta$-cell death in type 1 and type 2 diabetes: many differences, few similarities," Diabetes, vol. 54, Supplement 2, pp. S97-S107, 2005.
[5] A. Berbudi, J. Ajendra, A. P. Wardani, A. Hoerauf, and M. P. Hübner, "Parasitic helminths and their beneficial impact on type 1 and type 2 diabetes," Diabetes/Metabolism Research and Reviews, vol. 32, no. 3, pp. 238-250, 2016.

[6] A. Rabinovitch, W. L. Suarez-Pinzon, O. Sorensen, R. C. Bleackley, R. F. Power, and R. V. Rajotte, "Combined therapy with interleukin-4 and interleukin-10 inhibits autoimmune diabetes recurrence in syngeneic islet-transplanted nonobese diabetic mice. Analysis of cytokine mRNA expression in the graft," Transplantation, vol. 60, no. 4, pp. 368-374, 1995.

[7] A. Espinoza-Jimenez, A. N. Peon, and L. I. Terrazas, "Alternatively activated macrophages in types 1 and 2 diabetes," Mediators of Inflammation, vol. 2012, Article ID 815953, 10 pages, 2012.

[8] A. Cooke, P. Tonks, F. M. Jones et al., "Infection with Schistosoma mansoni prevents insulin dependent diabetes mellitus in non-obese diabetic mice," Parasite Immunology, vol. 21, no. 4, pp. 169-176, 1999.

[9] K. A. Saunders, T. Raine, A. Cooke, and C. E. Lawrence, "Inhibition of autoimmune type 1 diabetes by gastrointestinal helminth infection," Infection and Immunity, vol. 75, no. 1, pp. 397-407, 2007.

[10] Q. Liu, K. Sundar, P. K. Mishra et al., "Helminth infection can reduce insulitis and type 1 diabetes through CD25- and IL-10independent mechanisms," Infection and Immunity, vol. 77, no. 12, pp. 5347-5358, 2009.

[11] M. P. Hubner, J. T. Stocker, and E. Mitre, "Inhibition of type 1 diabetes in filaria-infected non-obese diabetic mice is associated with a T helper type 2 shift and induction of FoxP $3^{+}$ regulatory T cells," Immunology, vol. 127, no. 4, pp. 512-522, 2009.

[12] R. S. Peres, F. Chiuso-Minicucci, L. C. da Rosa et al., "Previous contact with Strongyloides venezuelensis contributed to prevent insulitis in MLD-STZ diabetes," Experimental Parasitology, vol. 134, no. 2, pp. 183-189, 2013.

[13] A. Espinoza-Jiménez, I. Rivera-Montoya, R. CárdenasArreola, L. Morán, and L. I. Terrazas, "Taenia crassiceps infection attenuates multiple low-dose streptozotocininduced diabetes," Journal of Biomedicine and Biotechnology, vol. 2010, Article ID 850541, 11 pages, 2010.

[14] S. Gordon, "Alternative activation of macrophages," Nature Reviews Immunology, vol. 3, no. 1, pp. 23-35, 2003.

[15] S. Gordon and F. O. Martinez, "Alternative activation of macrophages: mechanism and functions," Immunity, vol. 32, no. 5, pp. 593-604, 2010.

[16] L. I. Terrazas, D. Montero, C. A. Terrazas, J. L. Reyes, and M. Rodríguez-Sosa, "Role of the programmed death-1 pathway in the suppressive activity of alternatively activated macrophages in experimental cysticercosis," International Journal for Parasitology, vol. 35, no. 13, pp. 1349-1358, 2005.

[17] T. Kreider, R. M. Anthony, J. F. Urban Jr., and W. C. Gause, "Alternatively activated macrophages in helminth infections," Current Opinion in Immunology, vol. 19, no. 4, pp. 448-453, 2007.

[18] F. O. Martinez, L. Helming, and S. Gordon, "Alternative activation of macrophages: an immunologic functional perspective," Annual Review of Immunology, vol. 27, pp. 451-483, 2009.

[19] J. L. Reyes and L. I. Terrazas, "The divergent roles of alternatively activated macrophages in helminthic infections," Parasite Immunology, vol. 29, no. 12, pp. 609-619, 2007. 
[20] P. Zaccone, O. Burton, N. Miller, F. M. Jones, D. W. Dunne, and A. Cooke, "Schistosoma mansoni egg antigens induce Treg that participate in diabetes prevention in NOD mice," European Journal of Immunology, vol. 39, no. 4, pp. 1098-1107, 2009.

[21] P. Zaccone, Z. Fehérvári, F. M. Jones et al., "Schistosoma mansoni antigens modulate the activity of the innate immune response and prevent onset of type 1 diabetes," European Journal of Immunology, vol. 33, no. 5, pp. 1439-1449, 2003.

[22] P. Zaccone, O. T. Burton, S. Gibbs et al., "Immune modulation by Schistosoma mansoni antigens in NOD mice: effects on both innate and adaptive immune systems," Journal of Biomedicine and Biotechnology, vol. 2010, Article ID 795210, 11 pages, 2010.

[23] M. E. Lund, B. A. O'Brien, A. T. Hutchinson et al., "Secreted proteins from the helminth Fasciola hepatica inhibit the initiation of autoreactive $\mathrm{T}$ cell responses and prevent diabetes in the NOD mouse," PLoS One, vol. 9, no. 1, article e86289, 2014.

[24] D. Zheng, Y. Wang, Q. Cao et al., "Transfused macrophages ameliorate pancreatic and renal injury in murine diabetes mellitus," Nephron Experimental Nephrology, vol. 118, no. 4, pp. e87-e99, 2011.

[25] S. Okizaki, Y. Ito, K. Hosono et al., "Suppressed recruitment of alternatively activated macrophages reduces TGF- $\beta 1$ and impairs wound healing in streptozotocin-induced diabetic mice," Biomedicine \& Pharmacotherapy, vol. 70, pp. 317-325, 2015.

[26] E. Peranzoni, S. Zilio, I. Marigo et al., "Myeloid-derived suppressor cell heterogeneity and subset definition," Current Opinion in Immunology, vol. 22, no. 2, pp. 238-244, 2010.

[27] D. I. Gabrilovich and S. Nagaraj, "Myeloid-derived suppressor cells as regulators of the immune system," Nature Reviews Immunology, vol. 9, no. 3, pp. 162-174, 2009.

[28] L. Gómez-García, I. Rivera-Montoya, M. Rodríguez-Sosa, and L. I. Terrazas, "Carbohydrate components of Taenia crassiceps metacestodes display Th2-adjuvant and anti-inflammatory properties when co-injected with bystander antigen," Parasitology Research, vol. 99, no. 4, pp. 440-448, 2006.

[29] C. A. Terrazas, F. Sánchez-Muñoz, A. M. Mejía-Domínguez et al., "Cestode antigens induce a tolerogenic-like phenotype and inhibit LPS inflammatory responses in human dendritic cells," International Journal of Biological Sciences, vol. 7, no. 9, pp. 1391-1400, 2011.

[30] T. Szkudelski, "The mechanism of alloxan and streptozotocin action in B cells of the rat pancreas," Physiological Research, vol. 50, no. 6, pp. 537-546, 2001.

[31] J. L. Reyes, C. A. Terrazas, J. Alonso-Trujillo, N. van Rooijen, A. R. Satoskar, and L. I. Terrazas, "Early removal of alternatively activated macrophages leads to Taenia crassiceps cysticercosis clearance in vivo," International Journal for Parasitology, vol. 40, no. 6, pp. 731-742, 2010.

[32] H. J. McSorley, J. P. Hewitson, and R. M. Maizels, "Immunomodulation by helminth parasites: defining mechanisms and mediators," International Journal for Parasitology, vol. 43, no. 3-4, pp. 301-310, 2013.

[33] A. Cooke, P. Zaccone, T. Raine, J. M. Phillips, and D. W. Dunne, "Infection and autoimmunity: are we winning the war, only to lose the peace?," Trends in Parasitology, vol. 20, no. 7, pp. 316-321, 2004.

[34] L. M. Kuijk and I. van Die, "Worms to the rescue: can worm glycans protect from autoimmune diseases?," IUBMB Life, vol. 62, no. 4, pp. 303-312, 2010.
[35] R. M. Maizels and H. J. McSorley, "Regulation of the host immune system by helminth parasites," Journal of Allergy and Clinical Immunology, vol. 138, no. 3, pp. 666-675, 2016.

[36] R. M. Maizels, E. J. Pearce, D. Artis, M. Yazdanbakhsh, and T. A. Wynn, "Regulation of pathogenesis and immunity in helminth infections," The Journal of Experimental Medicine, vol. 206, no. 10, pp. 2059-2066, 2009.

[37] A. Cooke, "Review series on helminths, immune modulation and the hygiene hypothesis: how might infection modulate the onset of type 1 diabetes?," Immunology, vol. 126, no. 1, pp. 12-17, 2009.

[38] Y. Ledesma-Soto, B. E. Callejas, C. A. Terrazas et al., "Extraintestinal helminth infection limits pathology and proinflammatory cytokine expression during DSS-induced ulcerative colitis: a role for alternatively activated macrophages and prostaglandins," BioMed Research International, vol. 2015, Article ID 563425, 17 pages, 2015.

[39] J. L. Reyes, A. F. Espinoza-Jiménez, M. I. González, L. Verdin, and L. I. Terrazas, "Taenia crassiceps infection abrogates experimental autoimmune encephalomyelitis," Cellular Immunology, vol. 267, no. 2, pp. 77-87, 2011.

[40] M. Becerra-Diaz and L. I. Terrazas, "Taenia crassiceps infection and its excreted/secreted products inhibit STAT1 activation in response to IFN- $\gamma$," International Journal for Parasitology, vol. 44, no. 9, pp. 613-623, 2014.

[41] N. Amdare, V. Khatri, R. S. Yadav, A. Tarnekar, K. Goswami, and M. V. Reddy, "Brugia malayi soluble and excretorysecretory proteins attenuate development of streptozotocininduced type 1 diabetes in mice," Parasite Immunology, vol. 37, no. 12, pp. 624-634, 2015.

[42] J. Ajendra, A. Berbudi, A. Hoerauf, and M. P. Hübner, "Combination of worm antigen and proinsulin prevents type 1 diabetes in NOD mice after the onset of insulitis," Clinical Immunology, vol. 164, pp. 119-122, 2016.

[43] Y. Osada, T. Fujiyama, N. Kamimura et al., "Dual genetic absence of STAT6 and IL-10 does not abrogate anti-hyperglycemic effects of Schistosoma mansoni in streptozotocin-treated diabetic mice," Experimental Parasitology, vol. 177, 2017.

[44] B. Yin, G. Ma, C. Y. Yen et al., "Myeloid-derived suppressor cells prevent type 1 diabetes in murine models," The Journal of Immunology, vol. 185, no. 10, pp. 5828-5834, 2010.

[45] H. Yi, C. Guo, X. Yu, D. Zuo, and X. Y. Wang, "Mouse

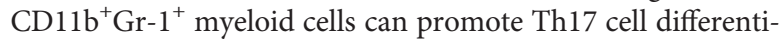
ation and experimental autoimmune encephalomyelitis," The Journal of Immunology, vol. 189, no. 9, pp. 4295-4304, 2012. 


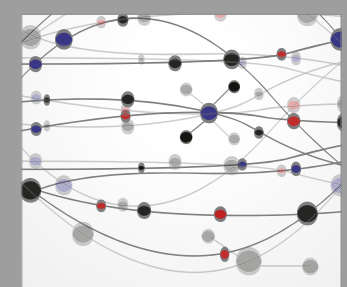

The Scientific World Journal
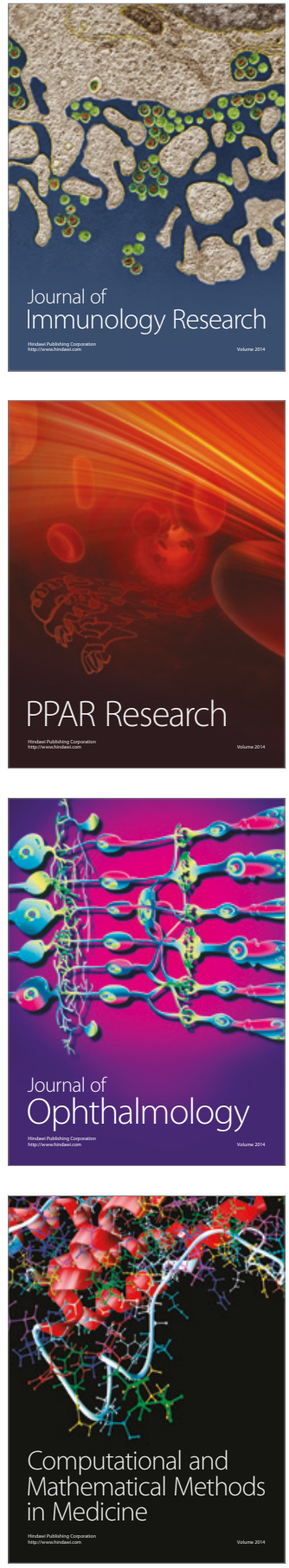

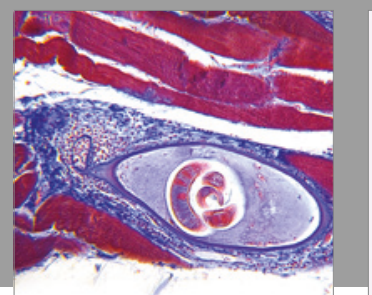

Gastroenterology Research and Practice
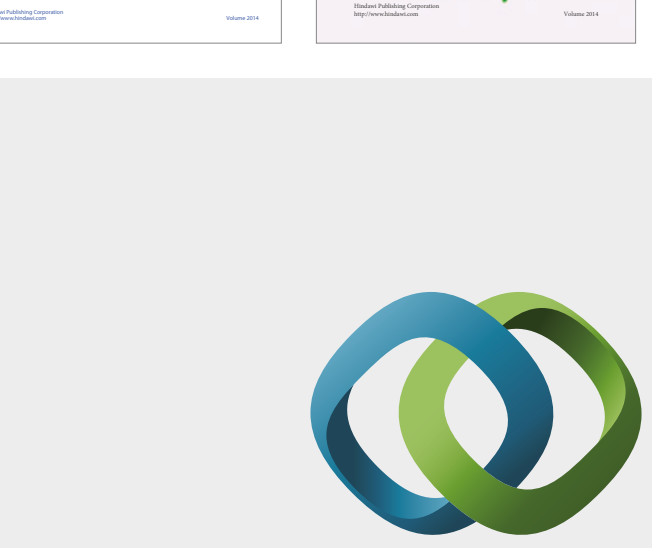

\section{Hindawi}

Submit your manuscripts at

https://www.hindawi.com
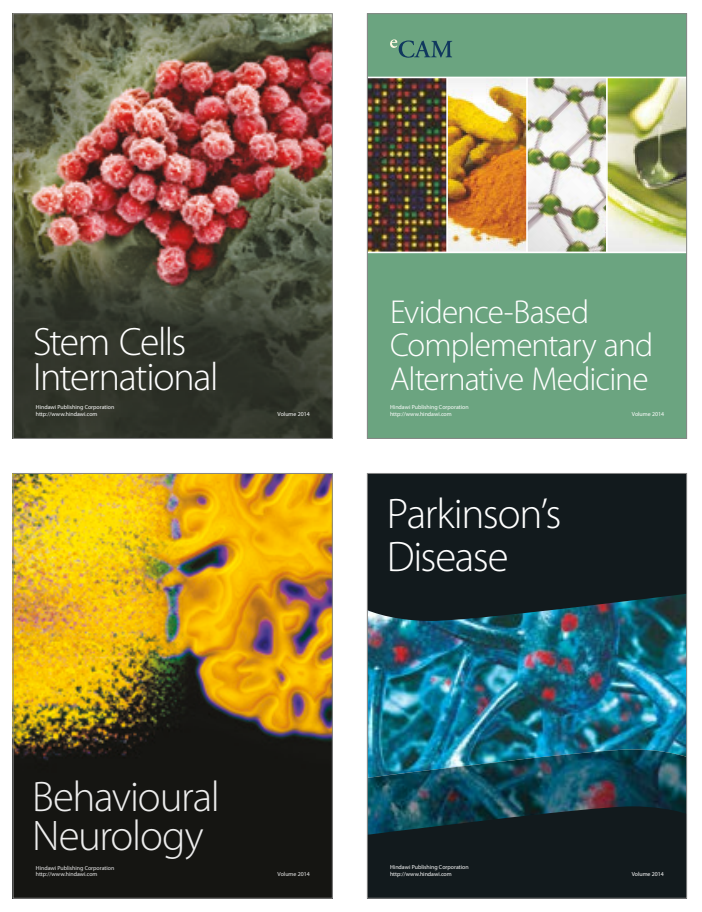
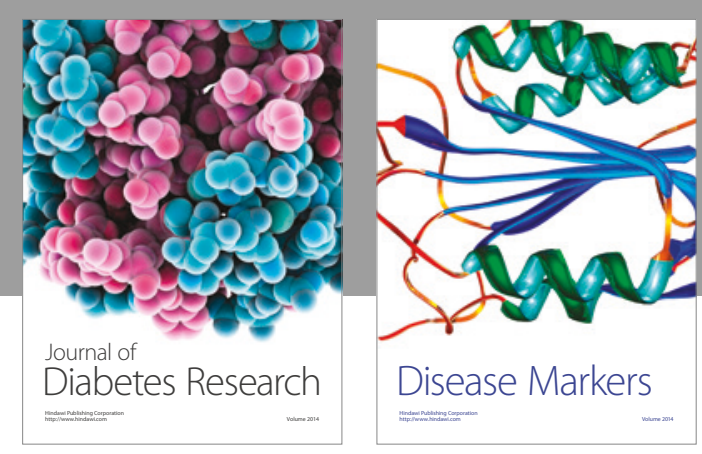

Disease Markers
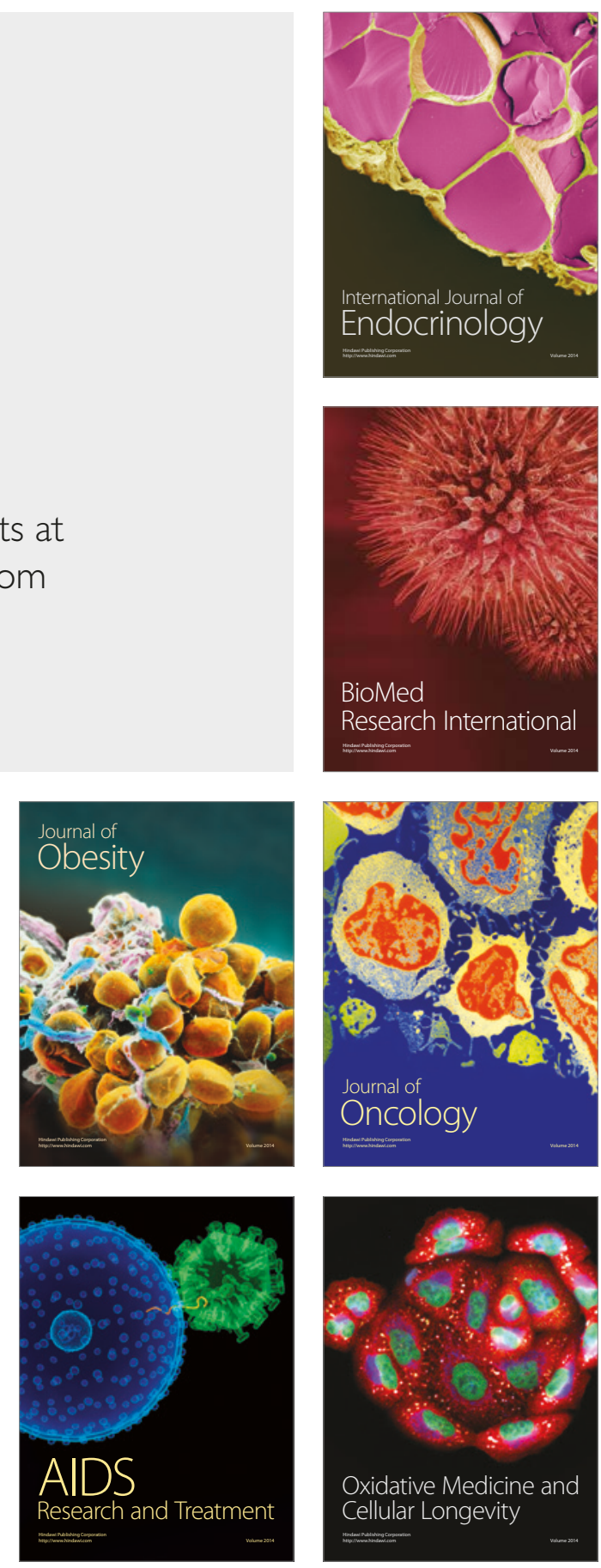